\title{
Exciton transport in molecular organic semiconductors boosted by transient quantum delocalization
}

\section{Samuele Giannini}

University College London https://orcid.org/0000-0002-1094-3921

\section{Wei-Tao Peng}

University College London

Lorenzo Cupellini

Università di Pisa https://orcid.org/0000-0003-0848-2908

\section{Daniele Padula}

University of Siena

\section{Antoine Carof}

Université de Lorraine https://orcid.org/0000-0003-1896-3094

Jochen Blumberger ( $\nabla$ j.blumberger@ucl.ac.uk)

University College London

\section{Article}

Keywords:

Posted Date: January 11th, 2022

DOI: https://doi.org/10.21203/rs.3.rs-1199006/v1

License: (1) This work is licensed under a Creative Commons Attribution 4.0 International License. Read Full License

Version of Record: A version of this preprint was published at Nature Communications on May 19th, 2022. See the published version at https://doi.org/10.1038/s41467-022-30308-5. 
Exciton transport in molecular organic semiconductors boosted by transient quantum delocalization

Samuele Giannini ${ }^{1,{ }^{\dagger},}$, Wei-Tao Peng ${ }^{1}$, Lorenzo Cupellini ${ }^{2}$, Daniele Padula ${ }^{3}$, Antoine Carof $^{4}$, and Jochen Blumberger ${ }^{1, *}$

${ }^{1}$ Department of Physics and Astronomy and Thomas Young Centre, University College London, London WC1E 6BT, UK.

${ }^{\dagger}$ Current address: Laboratory for Chemistry of Novel Materials, University of Mons, Mons 7000, Belgium

${ }^{2}$ Dipartimento di Chimica e Chimica Industriale, Universitá di Pisa, Via G. Moruzzi 13, 56124 Pisa, Italy

${ }^{3}$ Dipartimento di Biotecnologie, Chimica e Farmacia, Universitá di Siena, Via A. Moro 2, 53100, Siena, Italy.

${ }^{4}$ Laboratoire de Physique et Chimie Théoriques, CNRS, UMR No. 7019, Université de Lorraine, BP 239,54506 Vandoeuvre-lés-Nancy Cedex, France.

${ }^{*}$ Corresponding Authors, e-mail: samuele.giannini.16@ucl.ac.uk (S.G.), j.blumberger@ucl.ac.uk (J.B) 


\begin{abstract}
Designing molecular materials with very large exciton diffusion lengths would remove some of the intrinsic limitations of present-day organic optoelectronic devices. Yet, the nature of excitons in these materials is still not sufficiently well understood. Here we present Frenkel exciton surface hopping, a highly efficient method to propagate excitons through truly nano-scale materials by solving the timedependent Schrödinger equation coupled to nuclear motion. We find a clear correlation between diffusion constant and quantum delocalization of the exciton. In materials featuring some of the highest diffusion lengths to date, e.g. the non-fullerene acceptor Y6, the exciton propagates via a transient delocalization mechanism, reminiscent to what was recently proposed for charge transport. Yet, the extent of delocalization is rather modest, even in Y6, and found to be limited by the relatively large exciton reorganization energy. On this basis we chart out a path for rationally improving exciton transport in organic optoelectronic materials.
\end{abstract}

When an organic semiconductor (OS) absorbs light, electrons can be excited from the valence to the conduction band forming Coulombically bound electron-hole pairs. These excitations were described by Frenkel as energetic quasiparticles, which he named excitons. ${ }^{1,2}$ Frenkel excitons as we now call them can diffuse through the OS material or decay through various pathways, e.g., via fluorescence, intersystem crossing, phosphorescence, non-radiative transitions depending on the energetics of the system and its interactions. In several applications, in particular in organic photovoltaics (OPVs), OS materials with high diffusion lengths $L \propto \sqrt{D \tau}$ (where $D$ and $\tau$ are the exciton diffusion constant and lifetime, respectively) are highly desirable. Larger diffusion lengths mean larger domain sizes of the photoactive layer can be used, which would simplify device design and improve power conversion efficiency. ${ }^{3}$ While classical molecular OSs, suitable for OPV applications, have diffusion lengths of no more than 5-20 nm, ${ }^{4}$ several examples of new materials have recently been reported, termed non-fullerene acceptors (NFAs), that support longer range exciton transport, $20-47 \mathrm{~nm} .{ }^{5}$ The reason for this and the relations between molecular properties/packing and exciton transport are still not well understood. Establishing better computational approaches would help us understand the conditions in which exciton diffusion lengths are very large and beyond the values of today's best molecular materials.

In an extended system such as a molecular crystal, aggregate or polymer, excitons can be shared between molecular sites depending on the strength of the electronic interactions between the sites. As a consequence, the excited state spectrum of the material will differ from that of the isolated molecule. ${ }^{6,7}$ Recent theoretical and numerical advances in the related area of charge transport have provided strong evidence that charge carrier diffusion (i.e. mobility) in molecular materials is strongly correlated with the extent of charge carrier delocalization. ${ }^{8-10}$ One can expect that the same is true for excitons. Hence, computational modelling of materials with high exciton diffusivity need to take into account and be able to predict the extent of exciton delocalization in the material. This requires the development of efficient 
approaches that can be applied to system sizes large enough to fully accommodate delocalized excitons. Moreover, the method needs to take into account thermal vibrations that couple to the electronic interactions between the molecules (termed diagonal and off-diagonal electron-phonon coupling) as well as the so called "back reaction", namely the effect of the electronic interactions on the nuclear motion. Standard exciton hopping theories based on Fermi's Golden Rule, e.g., Marcus-Levich-Jortner, assume localized excitons and are thus expected to be of limited use for the modelling of materials with high exciton diffusion constants. ${ }^{11,12}$ Accurate quantum dynamics approaches like multi-layer multiconfigurational time dependent Hartree (ML-MCDTH) are arguably the gold standard for such problems - however they are typically limited to relatively small 1D model systems of a few hundred vibrational degrees of freedom. While they are excellent for benchmarking more approximate methods, they become computationally expensive, if not prohibitive, for truly nanoscale systems. ${ }^{13}$

In this work we introduce Frenkel exciton surface hopping (FE-SH), a computationally efficient atomistic non-adiabatic molecular dynamics method that strikes an optimal balance between predictive power and computational feasibility and that addresses the desirable criteria outlined above. In this method the excitonic wavefunction is represented as linear combinations of molecularly localized or quasi-diabatic Frenkel excitons interacting via excitonic couplings. This wavefunction is propagated across the material by solving the time-dependent Schrödinger equation coupled to the finite temperature motion of the nuclei, and feedback from the electronic to the nuclear degrees of freedom are incorporated by stochastic hops between the excitonic potential energy surfaces (see section Methods for details). A defining feature of FE-SH is that electronic structure calculations are only carried out for parametrization of the Frenkel exciton Hamiltonian matrix elements and their thermal fluctuations but not during FE-SH which makes the method highly efficient and amenable to large, nanoscale systems ( $>10 \mathrm{~nm}$ ), as opposed to other electronic structure-based methodologies present in the literature ${ }^{14-16}$. Conceptually, the method is similar to our previously presented fragment-orbital based surface hopping method (FOB-SH) for charge transport. ${ }^{17-19}$ An important difference with FOB-SH is that the short-ranged (exponentially decaying) electronic couplings and nuclear derivatives in FOB-SH are now replaced by the long-ranged excitonic couplings in FE-SH. Taking into account the long-ranged nature of the latter has been shown to be important in a recent coarse grained dynamics study of Frenkel excitons in polymer fibres. ${ }^{20,21}$ While our fully atomistic scheme can be relatively easily extended to include relaxation processes of Frenkel excitons such as exciton dissociation and recombination, in this work we place focus solely on singlet exciton transport.

Here we apply FE-SH to 5 different molecular organic crystals as shown in Figure 1, anthracene $(\mathrm{ANT})^{22}, \alpha$-sexithiophene (a6T) ${ }^{23}$, perylenetetracarboxylic diimide (PDI) ${ }^{24}$, dicyanovinyl-capped S,Nheteropentacene (DCVSN5) ${ }^{25}$, and $\mathrm{Y}^{26}{ }^{26}$. These systems are chosen to cover a wide range of diffusive regimes as well as for their relevance in organic optoelectronics. ANT has been very well studied ex- 
perimentally ${ }^{27-29}$ and computationally ${ }^{30,31}$ providing a good test case for our new FE-SH methodology. Polycrystalline a6T was recently shown to support exciton transport and dissociation without the presence of an acceptor material making it a good candidate for development of OPV homojunctions. ${ }^{32}$ It is also a good model system for thiophene-based polymeric light absorbers and electron donors in OPV heterojunctions. ${ }^{20}$ DCVSN5 is of interest because of the high exciton diffusion constant predicted for this material ${ }^{30}$ and because it was used as an electron donor in fullerene-based heterojunctions with moderate power conversion efficiency (about 6.5\%). ${ }^{30,33}$ Finally, we study two non-fullerene acceptor materials ${ }^{34}$, PDI as a representative of the chemically highly tunable class of perylene diamides ${ }^{35}$, as well as $\mathrm{Y}^{26,36}$. The latter has an intriguing chemical structure composed of alternating acceptor and donor moeities in the form A-DAD-A and it was used as the acceptor material in OPV devices with record-breaking power conversion efficiencies $>18 \%{ }^{36,37}$.

\section{Results}

The FE-SH method, presented in detail in section Methods, implements the excitonic time-dependent Schrödinger equation (Eq. 4) using the time-dependent Frenkel exciton Hamiltonian Eq. 2. In the following we investigate two key assumptions of our approach before presenting applications of FE-SH to molecular crystals. First, we establish that the Frenkel exciton Hamiltonian provides a suitable description of the electronic transitions in the systems studied. Second, we show that the total excitonic couplings between these states is well approximated by the Coulomb contribution only, similarly as in biological photosystems. ${ }^{38,39}$ The latter observation is of high practical significance allowing us to apply to method to truly nanoscale systems with little loss in accuracy and at moderate computational cost (details on the calculations presented below are given in the Methods section).

$\mathbf{S O} \rightarrow \mathbf{S 1}$ electronic transitions. We consider at first the S1 excited state of the isolated molecules and analyse the Natural Transition Orbitals (NTOs), shown in Figure 1. The NTOs offer a useful way of visualizing which orbitals gives the largest contribution to a given single-particle excitation. For all systems we can see that the NTOs with the largest contributions to the $\mathrm{S} 0 \rightarrow \mathrm{S} 1$ electronic transition resemble very closely the highest occupied molecular orbital (HOMO) and lowest unoccupied molecular orbital (LUMO) of the single molecules. Moreover, the second lowest singlet excited state, S2, is at least $0.5 \mathrm{eV}$ above $\mathrm{S} 1$ in all systems (values given in Supplementary Table 1). This implies that the low-energy tail of the lowest intra-molecular excitation band in the crystal is well described by a linear combination of the intra-molecular S1 states, and the latter, to a good approximation, by HOMO-LUMO transitions, attesting to the validity of the Frenkel exciton picture. Potentially lower-lying excited states, e.g., intermolecular charge transfer states may exist but are not considered here as focus is placed solely on the transport dynamics of Frenkel exciton states. Charge transfer states could be included by expansion of 
the state space of our electronic Hamiltonian, which is, however, beyond the scope of the present work. ${ }^{16}$

Excitonic Couplings. Next we turn to the calculation of excitonic couplings between the Frenkel states $k$ and $l, V_{k l}$ (in the following we skip the molecular indices $k, l$ for convenience). $V$ is typically written as a sum of a long-range or Coulombic part, $V^{\text {Coulomb }}$, and a short-range part, $V^{\text {short }}$, accounting for exchange, overlap and electronic polarization contributions, $V=V^{\text {Coulomb }}+V^{\text {short }} \cdot{ }^{40}$ In applications of FE-SH to nanoscale systems a very large number of excitonic couplings need to be calculated on-the-fly (typically $>10^{9}$ per trajectory) which mandates the use of an ultrafast coupling estimator. We do this by approximating $V$ by the long-range part $V^{\text {Coulomb }}$ (Eq. 10) and the latter by $V^{\operatorname{TrESP}}$ (Eq. 11) using frozen transition electrostatic potential (ESP) charges in place of transition densities, $V \approx V^{\text {Coulomb }} \approx V^{\text {TrESP }}{ }^{38,41}$ These two approximations introduce little error as shown in Table 1 and Figure 2. We find that $V^{\text {Coulomb }}$ obtained from the S1 excitation of the isolated molecules is very close to the total coupling $V$ (Figure 2a, mean relative unsigned error (MRUE) $\approx 6.6 \%$ ). $V$ accounts for all short- and long-range effects and was obtained by diabatization of the electronic excitations of molecular dimers using the multi-state fragment excitation energy difference-fragment charge difference method (MS-FED-FCD) in combination with TDDFT ${ }^{42-44}$. MS-FED-FCD, in turn, agrees well with available excitonic coupling from spin component scaled approximate coupled cluster theory (SCS-CC2), $27.9 \mathrm{meV}$ and $26 \mathrm{meV}$ for pair $P_{b}$ of anthracene ${ }^{11}$. Thus, our results indicate that the short-range coupling $V^{\text {short }}$ is of little importance even for the closely packed organic molecular solids studied here and can be neglected to a good approximation. A detailed discussion on this conclusion is given in the Supplementary Information and the importance of adopting a multi-state diabatization procedure to recover accurate excitonic couplings between (maximally) localized Frenkel exciton states is shown in Supplementary Figs. 1 and 2 and discussed in Methods.

In addition to the above, we find that $V^{\text {Coulomb }}$ is well approximated by $V^{\operatorname{TrESP}}$ (Figure 2b, MRUE $=7.0 \%)$. Moreover, the thermal fluctuations of $V^{\text {Coulomb }}$ along molecular dynamics trajectories with the transition densities updated at every MD step are very well reproduced by $V^{\text {TrESP }}$ with frozen transition charges (see Supplementary Figs. 3 and 4 and related discussion). These results are very significant because the calculation of $V^{\operatorname{TrESP}}$ is orders of magnitude faster than for $V^{\text {Coulomb }}$ allowing us to apply FE-SH to very large, nano-scale systems. Moreover it permits including excitonic couplings beyond nearest neighbour pairs, which can be significant due to their long-range nature $\left(V \propto r^{-3}\right)$ and important for an accurate estimation of exciton diffusion constants in certain systems, as shown previously. ${ }^{20,21}$ Interestingly, the common point dipole approximation (PDA) to $V^{\text {Coulomb }} V^{\text {PDA }}$ (Eq. 8 in Supplementary information), is found to break down for all molecules except ANT suggesting caution in the application of (dipoles based) Förster theory to these materials (see Supplementary Fig. 5). Further discussion of excitonic coupling calculations is given in the Supplementary Infomation.

Exciton transport mechanism from FE-SH non-adiabatic molecular dynamics. The exciton wave- 
function, $\Psi(t)$ (Eq. 3), is propagated in time across the $a-b$ planes of ANT, a6T, PDI and along a system composed of 5 and 4 columnar stacks for DCVSN5 and Y6, respectively, oriented along the $a$-direction (each system contains about 300 molecules). Notably, our FE-SH methodology obeys a number of desirable properties including energy conservation, internal consistency and detailed balance in the limit of long simulation times. ${ }^{18,19,45}$ We refer to the Methods section (and Supplementary Infomation) for full simulation details.

In the following we focus on the transport scenario in two systems that exhibit the lowest (ANT) and highest (DCVSN5) exciton delocalization of the 5 systems investigated. For ANT we find that the exciton remains localized on a single molecule for most of the time, as depicted by a value of the inverse participation ratio (IPR, Eq. 8) that is close to unity (Figure 3a, bottom panel). Thermal fluctuations occasionally lead to short-lived electronic excitations within the excitonic band (peaks in Figure 3a middle and upper panel) accompanied by transient delocalization over a nearest neighbour (increase in IPR to values of up to two, bottom panel in Figure 3a ). This is followed by de-excitation and concomitant re-localization of the exciton to either the neighbouring molecule, in which case the exciton becomes displaced, or back to the original molecule. Hence, our simulations show that ANT is an example for mostly nearest-neighbour hopping of a fully localized exciton.

This is no longer the case for the more diffusive system DCVSN5. The IPR is mostly about 2, i.e., the exciton is delocalized over 2 molecules on average (Figure 3b, bottom panel). Excitations to higher-lying states within the excitonic band are more frequent than for ANT (Figure 3b, upper and middle panels) and the transient expansions of the exciton are larger leading to delocalization over about 5-12 molecules. Importantly, the transient exciton delocalization facilitates coherent exciton transfer to molecules that are 3-4 lattice spacings apart $(\approx 20-30 \AA)$ permitting much larger spatial displacements than in ANT. In other words, in DCVSN5 exciton transfer is no longer bound to nearest neighbour hops, as was the case for ANT, but can occur across several molecules at a time, i.e. coherently, resulting in a boost of the diffusion constants, as quantified below. Y6 exhibits a similar transient delocalization mechanism and the transport scenario in the other materials studied is between the two limiting cases described.

Exciton diffusion constants. The mean squared displacement (MSD) of the exciton wavefunction averaged over 600 FE-SH trajectories is shown in Figure 4b-f for each system. We find that the MSD increases rapidly at short times ( $<100 \mathrm{fs}$ ) followed by a more shallow and approximately linear increase at longer times. The initial rapid increase is due to electronic relaxation of the initially fully localized exciton (which is a linear combination of eigenstates of the excitonic Hamiltonian Eq. 2) to states at the low energy tail of the excitonic band (see Methods for details). While the short-time relaxation dynamics within the excitonic band formed by the Frenkel states depends on the choice of the initial exciton wavefunction, the long-time diffusive dynamics is the same for different initial states. ${ }^{46,47}$ The linear increase of MSD at longer times is characteristic of Einstein diffusion and this is the regime for 
which we extract the diffusion tensor Eq. 6 (dashed lines in 4b-f). The diffusion constants along different crystallographic directions are well converged with regard to system size (see Supplementary Figure 8a). They span two orders of magnitude in the materials studied, they are highest in the direction of highest excitonic coupling, and exhibit a clear correlation with the average exciton delocalization (IPR), see Figure 4a. The latter quantity is also well converged for the supercells considered in this work (see Supplementary Figure 8b).

The magnitude and anisotropy of the computed diffusion constants are in good agreement with available experimental data. For ANT we obtain $D_{b}=3.3 \times 10^{-3} \mathrm{~cm}^{2} \mathrm{~s}^{-1}$ and $D_{a}=0.77 \times 10^{-3} \mathrm{~cm}^{2} \mathrm{~s}^{-1}$ compared with $D_{b}=5.0 \times 10^{-3} \mathrm{~cm}^{2} \mathrm{~s}^{-1}$ and $D_{a}=1.8 \times 10^{-3} \mathrm{~cm}^{2} \mathrm{~s}^{-1}$ estimated using the experimentally measured diffusion lengths, $L_{\text {exp }}$ (see Table 2). For this system, both diffusion constants obtained from FE-SH are slightly underestimated compared to experiments, similarly as in previous calculations by Elstner and co-workers who employed Boltzmann-corrected Ehrenfest dynamics for this system. ${ }^{31}$ A possible reason is the neglect of nuclear tunneling effects in our simulations, which can become significant for large activation barriers for the excitation energy transfer process, like in ANT. Another possible explanation is the absence of mixing between Frenkel exciton and charge transfer states (FE-CT) in present simulations, which, for some acenes, is known to enhance singlet exciton diffusion as a consequence of the larger exciton dispersion ${ }^{48}$. For an extended discussion of other computational methods applied to this system, including rate theory, see Supplementary Infomation and Supplementary Table 3.

A similarly good agreement with experiments is obtained at the higher end of diffusion constants, $D=150 \times 10^{-3} \mathrm{~cm}^{2} \mathrm{~s}^{-1}$ for Y6 from FE-SH compared with an estimated $D=54 \times 10^{-3} \mathrm{~cm}^{2} \mathrm{~s}^{-1}$ from experimentally determined diffusion lengths. ${ }^{5}$ For this system, measurements were carried out for thin film samples rather than single crystals, which could explain the somewhat larger computed $D$ compared to experiment. We also mention that wavefunction delocalization and diffusion constant of Y6 from the present simulations, although in good agreement with experiments, might constitute a lower limit as a more extended structural model in $b$ and $c$ directions might slightly increase the IPR and exciton diffusion in this 3D-like reticular system. The fact that the diffusion constant in Y6 is one order of magnitude larger than in ANT supports the picture from FE-SH simulation of a diffusion mechanism that is qualitatively different and more efficient than nearest neighbour hopping, similarly to what was found in Figure $3 \mathrm{~b}$ for DCVSN5. For the latter and other materials, to the best of our knowledge, no experimental estimates for exciton diffusion constants are available but qualitative comparisons to exciton diffusion lengths can be made which are again rather favourable, see Supplementary Infomation for a discussion. By using Ehrenfest dynamics coupled to a model Hamiltonian supplemented with effective local and non-local exciton-phonon coupling interactions, Ref. ${ }^{30}$ estimated an exciton diffusion constant of more than $1 \mathrm{~cm}^{2} \mathrm{~s}^{-1}$ for DCVSN5, which is much higher than what we found in this work. ${ }^{30} \mathrm{We}$ identified several contributing factors that might have led to such a large diffusion constant; electronic 
decoherence in the Ehrenfest approach is missing which is likely to result in an overestimation of the population of more delocalized higher-lying excited states at longer timescales; excitonic couplings are overestimated if only two 2 adiabatic electronic states are included in the diabatization procedure, as shown in Supplementary Fig. 2; neglect of excitonic couplings beyond nearest neighbours leads to slightly enhanced delocalized states at the bottom of the excitonic band, see Supplementary Figs. 9 and 10 and related discussion. Boltzmann-corrected Ehrenfest dynamics, as applied successfully to anthracene by Kranz et al. ${ }^{31}$, addresses some of the issues and gives a lower diffusion constant for ANT in better agreement with the value reported in this work (see Supplementary Table 3).

\section{Discussion}

Exciton vs Charge transport. Exciton diffusion and charge carrier transport are essential processes underpinning the function of organic optoelectronic devices. Yet, how does the diffusivity and delocalization of excitons compare to the ones of charge carriers? In the following we investigate this question by comparing the diffusion constants and IPRs for exciton transport with the values we obtained previously for charge transport using a similar surface hopping non-adiabatic molecular dynamics approach (termed FOB-SH) ${ }^{8,9}$. The results can only be indicative, as we compare exciton and charge diffusion constants for different sets of molecules, except in case of ANT where both values are available. To this end we plot in Figure $5 \mathrm{a}$ the exciton and charge diffusion constants along specific directions in the crystals as a function of the barrier height for (hypothetical) site-to-site exciton or charge transfer along this direction, $\Delta A^{\ddagger}$. In the 2-state harmonic approximation and for vanishing free energy difference between the sites of the crystal, which is typically a good approximation for organic crystals, the latter takes the form ${ }^{49,50}$

$$
\Delta A^{\ddagger}=\frac{\lambda}{4}-\left\langle\left|H_{k l}\right|^{2}\right\rangle^{1 / 2}+\frac{1}{\lambda}\left\langle\left|H_{k l}\right|^{2}\right\rangle
$$

where $H_{k l}$ is the excitonic coupling $\left(H_{k l}=H_{k l}^{\mathrm{XT}}\right)$ or electronic coupling for charge transfer $\left(H_{k l}=\right.$ $H_{k l}^{\mathrm{HT}}\left(H_{k l}^{\mathrm{ET}}\right)$ for hole (electron) transfer), and $\lambda$ is the reorganization energy for exciton or hole (electron) transfer. Large values of $\Delta A^{\ddagger}$ compared to $k_{\mathrm{B}} T$ are characteristic of small polaron hopping and small or vanishing values for delocalized polaronic transport. Note that the barrier height Eq. 1 is only defined for $\left\langle\left|H_{k l}\right|^{2}\right\rangle^{1 / 2}<\lambda / 2$; for larger coupling values the barrier disappears and becomes a minimum. ${ }^{49,50}$ We find that the data for exciton diffusion join the trend for charge diffusion rather well (data in blue and green in Figure 5a) - for both species the logarithm of the diffusion constant increases approximately linearly with decreasing barrier height, in accord with rate theories. The corresponding average IPR of exciton or charge carrier is shown in Figure 5b. Again, the data for excitons join the trend for charges except for systems with vanishing barrier height. Clearly, for barriers $>2 k_{\mathrm{B}} T \approx 50 \mathrm{meV}$ (ANT and a6T) the exciton 
is almost fully localized (as seen before in Figure 4a), whereas for barriers $<k_{\mathrm{B}} T$ (PDI, DCVSN5, Y6) delocalization occurs.

According to the data in Figure 5, excitons tend to have larger barrier heights, more localized wavefunctions and smaller diffusion constants than charge carriers, at least for the systems investigated here. Taking ANT as an example we found that the exciton is fully localized on a molecule whereas the electron hole is delocalized over 5 molecules on average ${ }^{9}$, see Supplementary Fig. 12 for a juxtaposition of the two wavefunctions. The main reason for this is that the reorganization energy (related to diagonal electron-phonon coupling) tends to be significantly larger, typically more than twice as large, for exciton transfer than for charge transfer. At the same time, the largest excitonic couplings are not significantly stronger than the largest electronic couplings for charge transfer (except for a6T), resulting in larger barrier heights (see Supplementary Table 4).

The large reorganization energies for exciton compared to charge transfer can be understood by looking at the NTOs that contribute most to the S1 transitions. Inspecting these orbitals (Figure 1) one can clearly observe that every bonding interaction in the HOMO-like NTO becomes an anti-bonding interaction in the LUMO-like NTO and vice versa. This causes large changes in the bond strengths and the bond lengths (up to $0.04 \AA$, Supplementary Fig. 7) when the geometry of the excited state is optimized resulting in large reorganization energies. In case of electron hole transport an electron is merely removed from the HOMO, which can be interpreted as a change of the bonding and anti-bonding interactions of the HOMO to no interactions. Therefore, the changes in bond lengths upon electron removal are smaller than for electronic excitation, typically half as large (up to $0.02 \AA$, Supplementary Figs. 7 and 11), resulting in smaller reorganization energies.

Besides reorganization energy, there are two other differences that contribute to the smaller exciton versus charge diffusivity, albeit to a lesser extent. The long-ranged nature of excitonic couplings tends to further increase localization of excitonic states at the bottom of the excitonic band when compared to results where only nearest neighbour couplings are included (see Supplementary Fig. 9), thus further decreasing exciton diffusion. This is consistent with what was recently found by Beljonne et al. in Ref. ${ }^{21}$ for short polymer fibers of P3HT. However, this effect is rather small for all excitonic systems investigated here. Another interesting difference concerns the root-mean-square fluctuations of excitonic and electronic couplings (see Table 1). The former are typically no larger than $10 \%$ of the mean values (except for ANT), whereas the latter are typically $50 \%$ of the mean value or more. ${ }^{9}$ The reason is that the excitonic couplings are only relatively weakly dependent on changes in geometry induced by lattice vibrations due to their Coulombic/long-range nature $\left(\propto r^{-3}\right)$ compared to the exponential sensitivity of electronic couplings. In the hopping regime (large barriers) coupling fluctuations are beneficial for the localized exciton to overcome the activation barrier and this could be the reason why charge transport in pMSB is somewhat faster than exciton transport in a6T although the two systems feature about the same 
barrier height and similar crystal packing arrangement.

The most diffusive excitonic systems investigated here, DCVSN5 and Y6, feature essentially barrierless diffusion owing to large excitonic couplings along the $P_{a}$ stacking direction (see Figure 1). Nonetheless, for DCVSN5 the diffusion constant in this direction is a factor of about 3 smaller than for charge transport in perylene crystals where barrier heights are also vanishingly small. Moreover, the exciton in DCVSN5 delocalizes over only about 2 molecules whereas the excess electron in perylene delocalizes over almost 12 molecules. ${ }^{9}$ The reason is that DCVSN5 forms a stacked structure giving rise to highly anisotropic, 1D exciton transport along the stacking direction, whereas perylene forms 2D layers that permit more isotropic, 2D electron transport. If only a 1D chain (along a) of the 2D layer of perylene is simulated the 1D charge diffusion constant and the wavefunction delocalization reduces to values that are very close to the ones for the exciton in DCVSN5 (Figure 5, black empty circles), showing that the dimensionality has a major impact on delocalization and diffusion of both excitons and charge carriers. The same conclusion holds as well when directly comparing exciton transport in DCVSN5 and Y6. Y6 shows more isotropic couplings within its reticular 3D-like structure and thus a higher diffusion constant than DCVSN5.

Design Rules The considerations above provide a road map for the design of molecular organic semiconductors with exciton diffusion constants that are beyond today's best materials (i.e., non-fullerene acceptors). We propose 3 "design rules" of decreasing importance. Rule 1 is to reduce reorganization energy to values of good charge transport, $<200 \mathrm{meV}$, while retaining high excitonic couplings so that the barrier Eq. 1 disappears. This is the case as soon as $\left\langle\left|H_{k l}\right|^{2}\right\rangle^{1 / 2}>\lambda / 2$, as, e.g., for hole transport in the high mobility organic crystal rubrene which supports extensive charge delocalization and high diffusion constants in the order of $0.5 \mathrm{~cm}^{2} \mathrm{~s}^{-1},{ }^{9}$ see Figure 5. Rule 2 is to increase the isotropy of excitonic coupling strength in the crystal, which will strongly increase delocalization of thermally accessible excitonic states resulting in the formation of multiple percolation pathways for barrierless diffusion. Once this is achieved one enters a regime where exciton delocalization and transport is no longer limited by reorganization energy or anisotropy but by the thermal fluctuations of excitonic couplings. Rule 3 is to reduce these fluctuations as much as possible as they contribute to wavefunction localization (note the difference with respect to the hopping regime where fluctuations are beneficial for transport ${ }^{9,21}$ ). Rule 3 is already better fulfilled by excitons than by charges owing to the smaller root-mean-square fluctuations of excitonic couplings compared the electronic couplings, as explained above (see Table 2). Each of the three rules aims to increase the delocalization of the excitonic states at the bottom of the excitonic band to which the exciton will relax via internal conversion before the diffusive regime sets in (disregarding here other possible relaxation channels such as charge transfer exciton formation, intersystem crossing or radiative recombination). Favourable sign combinations of excitonic couplings could form a fourth rule (as proposed for charge transport in Refs. ${ }^{51,52}$ ) and this issue, in connection with formation of $\mathrm{H}$ - and 
J-aggregation and their different spectroscopic signatures, ${ }^{6}$ deserves further investigation in forthcoming work.

In conclusion, we have introduced a highly efficient non-adiabatic molecular dynamics method, termed FE-SH, enabling the simulation of exciton transport in truly nanoscale organic semiconductors, including application-relevant NFA systems. The methodology makes no prior assumptions with regard to the degree of exciton (de)localization, it includes thermal fluctuations of excitonic couplings and site energies beyond the harmonic approximation. FE-SH is computationally fairly inexpensive because electronic structure calculations are not carried out during dynamics; the overhead compared to classical MD is only a factor of 4-5 per MD step (on a single core) for systems of a few hundred molecules. Therefore it is a powerful and practical tool to predict the mechanism and diffusion constants for exciton transport in molecular materials. Extensions of FE-SH are currently underway that will enable us to model the non-adiabatic processes that underpin the working principle of organic solar cells including exciton dissociation to interfacial charge transfer states, charge separation and recombination.

\section{Methods}

Frenkel exciton surface hopping (FE-SH). As stated in the main text, we consider singlet electronic excitations (XT) of a multichromophoric system. We restrict the treatment to a single excitonic band formed of local molecular excitations and we disregard other excited states such as triplet states or charge transfer states ${ }^{16,53}$. These can in principle be included in an extension of the model. The singlet electronic states of the system are then described in terms of local or quasi-diabatic molecular excitations (Frenkel excitons) and the resultant Hamiltonian, often referred to as Frenkel excitonic Hamiltonian, is written as

$$
\hat{H}^{\mathrm{XT}}=\sum_{k}^{M} \epsilon_{k}^{\mathrm{XT}}(t)\left|\phi_{k}^{\mathrm{XT}}\right\rangle\left\langle\phi_{k}^{\mathrm{XT}}\left|+\sum_{k}^{M} \sum_{l \neq k}^{M} H_{k l}^{\mathrm{XT}}(t)\right| \phi_{k}^{\mathrm{XT}}\right\rangle\left\langle\phi_{l}^{\mathrm{XT}}\right|,
$$

where $\left|\phi_{k}^{\mathrm{XT}}\right\rangle=\left|\phi_{k}^{\mathrm{XT}}(\mathbf{R}(t))\right\rangle$ represents the state with the exciton localized on molecule $k$ and all other molecules $l$ in the ground state, $\epsilon_{k}^{\mathrm{XT}}(\mathbf{R}(t))$ denotes the energy of that state, $H_{k l}^{\mathrm{XT}}(\mathbf{R}(t))$ denotes the intermolecular excitonic coupling between states $\left|\phi_{k}^{\mathrm{XT}}\right\rangle$ and $\left|\phi_{l}^{\mathrm{XT}}\right\rangle, M$ the number of molecules, and $\mathbf{R}(t)$ the time-dependent nuclear coordinates. We label these states with a superscript XT to distinguish them from the electron hole or excess electron states $(\phi)$ investigated previously ${ }^{8}$. The eigenstates of this Hamiltonian are the adiabatic excitonic states forming the excitonic band of the system. Writing the excitonic wavefunction as a linear combination of Frenkel excitons,

$$
\Psi(t)=\sum_{l=1}^{M} u_{l}(t)\left|\phi_{l}^{\mathrm{XT}}\right\rangle,
$$


and inserting this expression in the time-dependent Schrödinger equation, one obtains the time-evolution of the excitonic wavefunction within the excitonic band:

$$
i \hbar \dot{u}_{k}(t)=\sum_{l=1}^{M} u_{l}(t)\left(H_{k l}^{\mathrm{XT}}(\mathbf{R}(t))-i \hbar d_{k l}^{\mathrm{XT}}(\mathbf{R}(t))\right)
$$

where we have assumed that the quasi-diabatic excitonic states are orthogonal. This equation for exciton propagation is formally equivalent to the one used before for charge propagation in FOB-SH non-adiabatic dynamics ${ }^{17}$; Frenkel exciton states merely replace hole or excess electron states. The last term on the right hand side of Eq. 4 that includes the non-adiabatic coupling between the quasidiabatic states, $d_{k l}^{\mathrm{XT}}=\left\langle\phi_{k}^{\mathrm{XT}} \mid \dot{\phi}_{l}^{\mathrm{XT}}\right\rangle$, can be assumed to be negligibly small compared to excitonic coupling, $\left|i \hbar d_{k l}^{\mathrm{XT}}\right|<<\left|H_{k l}^{\mathrm{XT}}\right|$, and is neglected. We present detailed arguments for the validity of this approximation at the end of this section. In accord with Tully's fewest switches surface hopping algorithm, the nuclear degrees of freedom are propagated on one of the potential energy surfaces (PES) obtained by diagonalizing the Hamiltonian Eq. 2 and denoted as $E_{a}$ (" $a$ " for "active surface"). The nuclear motion couples to exciton motion via the dependences of all exciton Hamiltonian matrix elements on $\mathbf{R}(t)$, see Eq. 4 , resulting in diagonal and off-diagonal exciton-phonon coupling. The coupling in the reverse direction (also denoted "feedback") from the exciton to the nuclear motion is accounted for by transitions of the nuclear dynamics ("hops") from the PES of the active eigenstate $a, E_{a}$, to the PES of another eigenstate $j$ using Tully's surface hopping probability. ${ }^{54}$

Nuclear forces required to propagate the nuclear dynamics are calculated using the Hellmann-Feynman theorem. The force acting on nucleus $I$ on potential energy surface $E_{a}$ can be expressed as

$$
\mathbf{F}_{I, a}=-\nabla_{I} E_{a}=-\left[\mathbb{U}^{\dagger}\left(\nabla_{I} \mathbb{H}^{\mathrm{XT}}\right) \mathbb{U}\right]_{a a}
$$

where $\nabla_{I} \equiv \partial / \partial \mathbf{R}_{I}, \mathbb{H}^{\mathrm{XT}}$ is the Frenkel Hamiltonian Eq. 2 in matrix representation and $\mathbb{U}$ the unitary matrix diagonalizing $\mathbb{H}^{\mathrm{XT}}$. We refer to Ref. ${ }^{17}$ for an explicit derivation of Eq. 5. The nuclear derivatives of the diagonal elements, $\nabla_{I} H_{k k}^{\mathrm{XT}}=\nabla_{I}\left[\mathbb{H}^{\mathrm{XT}}\right]_{k k}$, are taken as the gradients of the classical force field potential used to calculate the site energies of excitonic state $k$ (see section "site energies" below). The off-diagonal gradients, $\nabla_{I} H_{k l}^{\mathrm{XT}}=\nabla_{I}\left[\mathbb{H}^{\mathrm{XT}}\right]_{k l}, k \neq l$, are taken as the gradients of the excitonic couplings evaluated in the TrESP approximation (see section "excitonic couplings" below), $\nabla_{I} H_{k l}^{\mathrm{XT}}=\nabla_{I} V_{k l}^{\mathrm{TrESP}}$, where $V_{k l}^{\mathrm{TrESP}}$ is given by Eq. 11 and they are calculated analytically.

As for charge transport simulation, the surface hopping algorithm needs to be supplemented with a number of important features so the method can be applied to the calculation of transport properties. $8,9,55$ These are decoherence correction, trivial crossing detection, elimination of spurious long-range exciton transfer and adjustment of the velocities in the direction of the non-adiabatic coupling vector in case of 
a successful surface hop. We refer to Ref. ${ }^{18,19}$ for a detailed description and discussion of the importance and the physical underpinnings of these additions to the original fewest switches surface hopping method. The above approach Eqs. 2-5 in combination with Tully's surface hopping probability defines our Frenkel exciton surface hopping method (FE-SH).

Diffusion tensor and IPR. Solving Eq. 4, one obtains the excitonic wavefunction as a function of time, $\Psi(t)$. This gives access to key dynamical properties, e.g. the diffusion tensor, the extent of localization or delocalization of the exciton as a function of time and the mechanism by which the excitonic wavefunction moves within the material. The (second rank) diffusion tensor components, $D_{\alpha \beta}$, can be obtained as the time derivative of the mean squared displacement along the nine Cartesian components $\left(\mathrm{MSD}_{\alpha \beta}\right)$,

$$
D_{\alpha \beta}=\frac{1}{2} \lim _{t \rightarrow \infty} \frac{\mathrm{dMSD}_{\alpha \beta}(t)}{\mathrm{d} t}
$$

where $\alpha, \beta$ denote the Cartesian coordinates $x, y, z$ and

$$
\begin{aligned}
\operatorname{MSD}_{\alpha \beta}(t) & =\frac{1}{N_{\text {traj }}} \sum_{n=1}^{N_{\text {traj }}}\left\langle\Psi_{n}(t)\left|\left(\alpha-\alpha_{0, n}\right)\left(\beta-\beta_{0, n}\right)\right| \Psi_{n}(t)\right\rangle \\
& \approx \frac{1}{N_{\text {traj }}} \sum_{n=1}^{N_{\text {traj }}} \sum_{k=1}^{M}\left|u_{k, n}\right|^{2}(t)\left(\alpha_{k, n}-\alpha_{0, n}\right)\left(\beta_{k, n}-\beta_{0, n}\right) .
\end{aligned}
$$

In Eq. 7, $\Psi_{n}(t)$ is the time-dependent excitonic wavefunction in trajectory $n, \alpha_{0, n}\left(\beta_{0, n}\right)$ are the initial positions of the excitonic wavefunction, $\alpha_{0, n}=\left\langle\Psi_{n}(0)|\alpha| \Psi_{n}(0)\right\rangle$, and the square displacements are averaged over $N_{\text {traj }}$ FE-SH trajectories. In the second equation the coordinates of the exciton are discretized and replaced by the center of mass of molecule $k$ in trajectory $n, \alpha_{k, n}$, and $\alpha_{0, n}=\sum_{k=1}^{M}\left|u_{k, n}\right|^{2}(0) \alpha_{k, n}(0)$, where $\left|u_{k, n}\right|^{2}(t)$ is the time dependent excitonic population of site $k$ in trajectory $n$ as obtained by solving Eq. 4 . The elements of the diffusion tensor, $D_{\alpha \beta}$, can be used to define the diffusion lengths ${ }^{4}, L_{\alpha \beta}=\sqrt{2 D_{\alpha \beta} \tau}$, where $\tau$ is the exciton lifetime, i.e., the time it takes for the exciton to relax to the ground state. The latter quantity can be obtained from, e.g., photo-luminescence experiments. ${ }^{4}$

We use the inverse participation ratio (IPR) as a measure to describe the delocalization of the excitonic wavefunction $\Psi(t)$,

$$
\operatorname{IPR}(t)=\frac{1}{N_{\text {traj }}} \sum_{n=1}^{N_{\text {traj }}} \frac{1}{\sum_{k=1}^{M}\left|u_{k, n}\right|^{4}(t)} .
$$

The numerical value of the IPR is about equal to the number of molecules the wavefunction is delocalized over. A similar definition is used to describe the delocalization of the adiabatic states or eigenstates of 
the Hamiltonian Eq. 2, $\psi_{i}^{\mathrm{XT}}(t)$,

$$
\operatorname{IPR}_{i}(t)=\frac{1}{N_{t r a j}} \sum_{n=1}^{N_{t r a j}} \frac{1}{\sum_{k=1}^{M}\left|U_{k i, n}\right|^{4}(t)}
$$

where $U_{k i, n}$ are the components of the eigenvector $i$ of the Hamiltonian Eq. 2 in trajectory $n$.

Excitation energies. We computed the excitation energies of ANT, a6T, PDI, DCVSN5 and Y6 single molecules with two different long range-separated hybrid functionals, CAM-B3LYP ${ }^{56}$ and $\omega$ B97X-D ${ }^{57}$. These functionals are commonly used for excitation energy calculations on organic molecules due to their correct asymptotic behaviour and balanced description of locally excited and charge transfer states. The basis set was fixed to $6-31 \mathrm{G}(\mathrm{d}, \mathrm{p})$ as commonly used in the literature for similar systems ${ }^{30,58,59}$. The vertical and adiabatic excitation energies as well as the reorganization energies, $\lambda^{\mathrm{XT}}$ (Eq. 12), for the lowest singlet excited state (S1) of ANT, a6T, PDI, DCVSN5 and Y6 are summarized in Supplementary Table 1. All quantum chemical calculations have been performed using the TDDFT implementation in the Gaussian16 software package ${ }^{60}$.

Excitonic couplings. The excitonic coupling between states $\left|\phi_{k}^{\mathrm{XT}}\right\rangle$ and $\left|\phi_{l}^{\mathrm{XT}}\right\rangle, H_{k l}^{\mathrm{XT}}$, is computed in the dimer approximation in vacuum. Molecules $k$ and $l$, with geometries taken from the condensed phase (crystal structure or FE-SH simulation), form the donor-acceptor pair and the two exciton states are denoted $\left|k^{*} l\right\rangle$ and $\left|k l^{*}\right\rangle$, respectively. The excitonic coupling in the dimer approximation is denoted $V_{k l}=\left\langle k^{*} l\left|\hat{H}_{\text {dimer }}\right| k l^{*}\right\rangle$. Reference excitonic couplings $V_{k l}$, shown in Figure 2, were calculated using a multi-state diabatization procedure MS-FED-FCD ${ }^{42,43,61}$. First, the adiabatic excited states of the donoracceptor pair is calculated followed by diabatization to localized, (maximally) diabatic excitonic states ${ }^{43}$. The excitonic coupling corresponds to the off-diagonal element between the locally excited (Frenkel) states and includes all long- and short-range contributions. We found that a 2-state adiabatic basis is not sufficient to retrieve completely localized states: in fact, an adiabatic state could be the combination of many diabatic states of both donor and acceptor. Moreover, charge transfer states can mix with Frenkel exciton states, and vice versa ${ }^{42,61}$. To overcome this difficulty and recover the coupling between completely de-mixed Frenkel exciton states - which form the state space for the Frenkel Hamiltonian in Eq. 2 - we included up to 20 excited states of the donor-acceptor systems to ensure a complete de-mixing between excitations of different nature and an optimal reconstruction of localized Frenkel exciton states and related couplings. We refer to Supplementary Figs. 1 and 2 and related discussion for a convergence analysis.

As explained in the main text, $V$ can be written in terms of a long-range or Coulomb term and a shortrange term, $V_{k l}=V_{k l}^{\text {Coulomb }}+V_{k l}^{\text {short } 40,62}$. The Coulomb term is defined by the Coulombic interaction 
between the transition densities of isolated donor and acceptor (singlet) states,

$$
V_{k l}^{\text {Coulomb }}=\int \mathrm{d} \mathbf{r} \int \mathrm{d} \mathbf{r}^{\prime} \rho_{k}^{T^{*}}\left(\mathbf{r}^{\prime}\right) \frac{1}{\left|\mathbf{r}-\mathbf{r}^{\prime}\right|} \rho_{l}^{T}(\mathbf{r})
$$

where $\rho_{k}^{T}$ and $\rho_{l}^{T}$ are the diagonal parts of the one-particle density matrix constructed from the ground and excited-state wave functions of isolated donor and acceptor. The transition densities of each molecule can be computed efficiently through an atomic orbital expansion in combination with configuration interaction singles (CIS) and TDDFT ${ }^{40}$. Note that transition densities are computed on isolated donor/acceptor in these calculations, as opposed to MS-FED-FCD calculations (where a calculation on the whole donoracceptor pair is performed). The total excitonic coupling as well as the Coulomb term Eq. 10 were computed within the TDDFT framework implemented in the Gaussian16 software package ${ }^{60,63}$ employing the same functionals and basis set as for the calculation of excitation and reorganisation energies below.

During FE-SH propagation, the transition densities are approximated by transition charges resulting in the following approximation for the Coulomb integral Eq. 10:

$$
V_{k l}^{\operatorname{TrESP}}=\sum_{A \in k}^{N} \sum_{B \in l}^{N} \frac{q_{A}^{T} q_{B}^{T}}{\left|\mathbf{r}_{A}-\mathbf{r}_{B}\right|},
$$

where the indices $A$ and $B$ run over the atoms of molecules $k$ and $l$, respectively, $q_{A}^{T}$, $q_{B}^{T}$ are the transition charges and $\mathbf{r}_{A}, \mathbf{r}_{B}$ the positions of atoms $A$ and $B$, respectively. Herein, we use the transition charges obtained from the fitting of the electrostatic potential (TrESP). TrESP charges are obtained as proposed by Renger et al. ${ }^{41}$, fitting the electrostatic potential generated by the transition density. When used in the FESH framework, TrESP charges are computed once for the isolated molecule in vacuum, prior production run, and then kept frozen along the trajectory (see Table 1, Figure 2 and Supplementary Figure 2 for a discussion on the accuracy of this approach). The error due to keeping the transition charges fixed along dynamics was investigated and found to be rather small (see discussion and related Supplementary Figs 3 and 4 in the Supplementary Information).

Site energies. The approach chosen for the calculation of site energies, that is, the diagonal elements of the excitonic Hamiltonian Eq. 2 is very similar to the one for hole or excess electron transport in FOB$\mathrm{SH}$. The potential energy of excitonic state $\phi_{k}^{\mathrm{XT}}$ is obtained from classical force fields, where molecules $k$ $(l \neq k)$ are described with force field parameters parametrized to the singlet excited (ground) state of the molecules as obtained from TDDFT calculations. In particular, the equilibrium distances for bonding interactions in the excited state are displaced with respect to the ones for the ground state to reproduce the exciton reorganization energy ${ }^{30,64}, \lambda^{\mathrm{XT}}$, from TDDFT calculations (see Supplementary Fig. 7). The 
latter is calculated using the four point scheme,

$$
\lambda^{\mathrm{XT}}=\left[E_{\mathrm{S} 1}\left(\mathbf{R}_{\mathrm{S} 0}\right)+E_{\mathrm{S} 0}\left(\mathbf{R}_{\mathrm{S} 1}\right)\right]-\left[E_{\mathrm{S} 1}\left(\mathbf{R}_{\mathrm{S} 1}\right)+E_{\mathrm{S} 0}\left(\mathbf{R}_{\mathrm{S} 0}\right)\right] .
$$

where $E_{\mathrm{S} 0}$ and $E_{\mathrm{S} 1}$ denote the potential energy of the ground state and $\mathrm{S} 1$ excited state, respectively, and $\mathbf{R}_{\mathrm{S} 0}$ and $\mathbf{R}_{\mathrm{S} 1}$ the nuclear coordinates at the minimum of the S0 and S1 potential energy surfaces.

FE-SH simulation Details. For each system, we built a series of supercells from the experimental crystallographic unit cell. The dimensions of the largest supercells generated are summarized in Supplementary Table 2. For ANT, a6T and PDI, the intra- and inter-molecular interaction terms for the ground state are taken from the Generalized Amber Force Field (GAFF) ${ }^{65}$. They describe well the structure and vibrational properties of acenes and conjugated systems. ${ }^{66}$ For DCVSN5 and Y6 we employed the parametrization proposed in Ref. ${ }^{30}$ and Ref. ${ }^{67}$, respectively. We refer to the latter references for a full description of the parametrization procedure and related validation.

These supercells were equilibrated in periodic boundary conditions, with all molecules in the ground state, for at least 250 ps in the NVT ensemble to a target temperature of $300 \mathrm{~K}$ using a Nosé-Hoover thermostat. This was followed by equilibration for at least 250 ps in the NVE ensemble. ${ }^{8,18}$ From the NVE trajectory an uncorrelated set of positions and velocities were chosen as starting configurations for FE-SH simulations. Molecules within a given region of the supercell were treated as electronically active, i.e., as molecular sites or fragments for construction of the Frenkel Hamiltonian Eq. 2, as previously done for charge transport with FOB-SH ${ }^{8,9}$. All other molecules of the supercell were treated electronically inactive and interacted with the active region only via non-bonded interactions. The number of electronically active molecules was ca. 300 for each system simulated as shown in Supplementary Fig. 8 (300 molecules for ANT, 312 for a6T, 336 for PDI, 340 for DCVSN5 and 328 for Y6). For ANT, a6T and PDI, we consider active molecules only within the $a-b$ plane, which is the plane where the strongest exciton interactions are present. The DCVSN5 and Y6 crystals form a different crystal structure compared to the aforementioned systems. DCVSN5 arranges in antiparallel $\pi$-stacked columns with a short intercolumnar distance, whereas $\mathrm{Y} 6$ forms a porous interpenetrating supramolecular structure (see Figure 1). The largest excitonic couplings for both systems are along the $\pi$-stacked pillars formed by the electron accepting and donating units of neighbouring molecules ( $a$ crystallographic direction). However, these systems exhibit also inter-columnar excitonic interactions in approximately perpendicular to $a$. To account for the inter-columnar interactions we include a number of columnar stacks in our model (5 for DCVSN5 and 4 for Y6) resulting in an electronically active region of tubular shape. Though, the lateral dimension of the tubular stacks is not sufficiently large for calculation of exciton diffusion constants in that direction. Thus, our simulations only yield the diffusion constant along the $\pi$-stacked pillars in these two materials. 
The initial excitonic wavefunction is chosen to be initially localized on a single active molecule $m$, $\Psi(t=0)=\phi_{m}^{\mathrm{XT}}$, where $\phi_{m}^{\mathrm{XT}}$ is the Frenkel exciton located on molecule $m$ and propagated in time according to the FE-SH algorithm described above in the NVE ensemble. The nuclear time step, $\Delta t$, was chosen as small as $0.01 \mathrm{fs}$ to prevent trivial crossings (which becomes more problematic for narrow excitonic bandwidth and localized excitonic wavefunction). Eq. 4 was integrated using the 4th order Runge-Kutta algorithm and a time step of $\delta t=\Delta t / 5$. For each system about $600 \mathrm{FE}-\mathrm{SH}$ trajectories of length of at least 1 ps were run for calculation of the diffusion tensor and IPR. The components of the MSD Eq. 7 were block averaged over 3 blocks (at least 200 trajectories each) for calculation of error bars (see Figure 4). The convergence of the diffusion constant with the number of electronically active molecules is shown in Supplementary Fig. 8. All simulations were carried out with our in-house implementation of FE-SH in the CP2K simulation package. ${ }^{68}$

Neglect of $d_{12}^{\mathrm{XT}}$ in Eq. 4. We consider a simple 2-site model with one electron on each site occupying either the ground state $\chi_{k}^{\mathrm{H}}$ or the excited state $\chi_{k}^{\mathrm{L}}, k$ the site index, $k=1,2$. In the initial state (1) the electron on site 1 is in the excited state and the electron on site 2 is in the ground state and vice versa for the final state (2). The corresponding wavefunctions are $\phi_{1}^{\mathrm{XT}}=2^{-1 / 2} \operatorname{det}\left(\chi_{1}^{\mathrm{L}}\left(r_{1}\right) \chi_{2}^{\mathrm{H}}\left(r_{2}\right)\right)$ and $\phi_{2}^{\mathrm{XT}}=2^{-1 / 2} \operatorname{det}\left(\chi_{1}^{\mathrm{H}}\left(r_{1}\right) \chi_{2}^{\mathrm{L}}\left(r_{2}\right)\right)$. It can be shown that the non-adiabatic coupling between the two states,

$d_{12}^{\mathrm{XT}}=\left\langle\phi_{1}^{\mathrm{XT}} \mid \dot{\phi}_{2}^{\mathrm{XT}}\right\rangle$, is given by $d_{12}^{\mathrm{XT}}=-\left(\left\langle\chi_{1}^{\mathrm{L}} \mid \dot{\chi}_{2}^{\mathrm{L}}\right\rangle\left\langle\chi_{2}^{\mathrm{H}} \mid \chi_{1}^{\mathrm{H}}\right\rangle+\left\langle\chi_{2}^{\mathrm{H}} \mid \dot{\chi}_{1}^{\mathrm{H}}\right\rangle\left\langle\chi_{1}^{\mathrm{L}} \mid \chi_{2}^{\mathrm{L}}\right\rangle\right)$, where we have assumed that the ground and excited states on the same site are orthogonal, $\left\langle\chi_{k}^{\mathrm{L}} \mid \chi_{k}^{\mathrm{H}}\right\rangle=0$. For multi-electron sites with the S1 excitation dominated by the HOMO-LUMO transition, the above expression with L the LUMO orbital and $\mathrm{H}$ the HOMO orbital becomes the leading terms of non-adiabatic coupling. According to this expression, the non-adiabatic coupling is the product of the time-dependent change in LUMO (HOMO) overlap on different molecules $k$ and $l$ times the HOMO (LUMO) overlap, also taken on molecules $k$ and $l$. Each of the two terms are exponentially decaying with distance and are very small at molecular separations compared to the leading (Coulombic) term of excitonic coupling. In fact, the situation for excitons is even more favourable than for hole or excess electron transfer, where it was shown that a similar approximation induces virtually no changes in the electron dynamics ${ }^{9}$. In the latter case the leading term to non-adiabatic coupling is proportional to the time-dependent change in LUMO (HOMO) overlap, only. For exciton transfer the additional HOMO (LUMO) overlap term decreases the non-adiabatic coupling between the two quasi-diabatic states further. Thus, we expect this approximation to hold for excitons even better than for hole or excess electron transfer.

\section{Additional Information}

Supplementary Information accompanies this paper. 


\section{Acknowledgement}

The authors would like to acknowledge Prof. David Beljonne and Prof. Benedetta Mennucci for useful discussions. S.G. would like to thank Dr. Orestis G. Ziogos and Dr. Matthew Ellis for important inputs on the classical force fields and wavefunction visualization tool respectively employed in this work. S.G. and P.W. were supported by the European Research Council (ERC) under the European Union, Horizon 2020 research and innovation programme (grant agreement no. 682539/SOFTCHARGE). Via our membership of the UK's HEC Materials Chemistry Consortium, which is funded by EPSRC (EP/L000202, EP/R029431), this work used the ARCHER UK National Supercomputing Service (http://www.archer.ac.uk) as well as the UK Materials and Molecular Modelling (MMM) Hub, which is partially funded by EPSRC (EP/P020194), for computational resources. We also acknowledge the use of the UCL Kathleen High Performance Computing Facility. D.P. acknowledges the Italian Ministry of Education, University, and Research (MIUR) for a Rita Levi Montalcini grant.

\section{Competing Interests}

The authors declare no competing interests.

\section{Author contributions}

S.G. developed the FE-SH code, with inputs from A.C.. S.G also performed most of the quantum chemical calculations, FE-SH parametrization and exciton dynamics for the systems investigated in this work (with inputs from J.B.). W.P. parametrized and simulated exciton diffusion in $\mathrm{Y} 6$ with inputs from D.P., S.G. and J.B. The MS-FED-FCD was developed by L.C., who also helped with exciton coupling calculations and data interpretation. J.B. supervised each step of the research and contributed with data interpretation. S.G. and J.B. designed the research and wrote the manuscript. All authors reviewed and discussed the manuscript. 
a

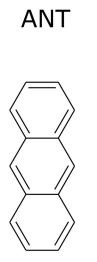

b

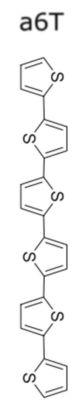

C

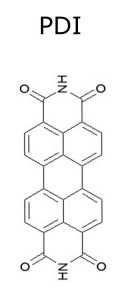

d

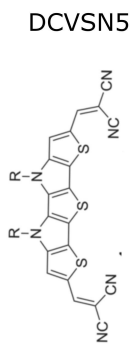

$\mathbf{e}$

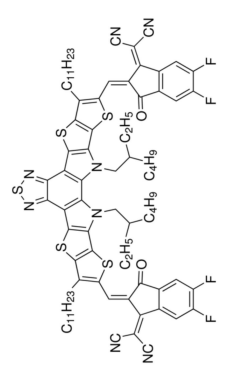

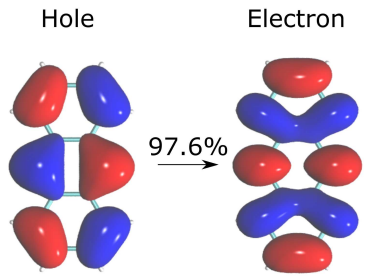
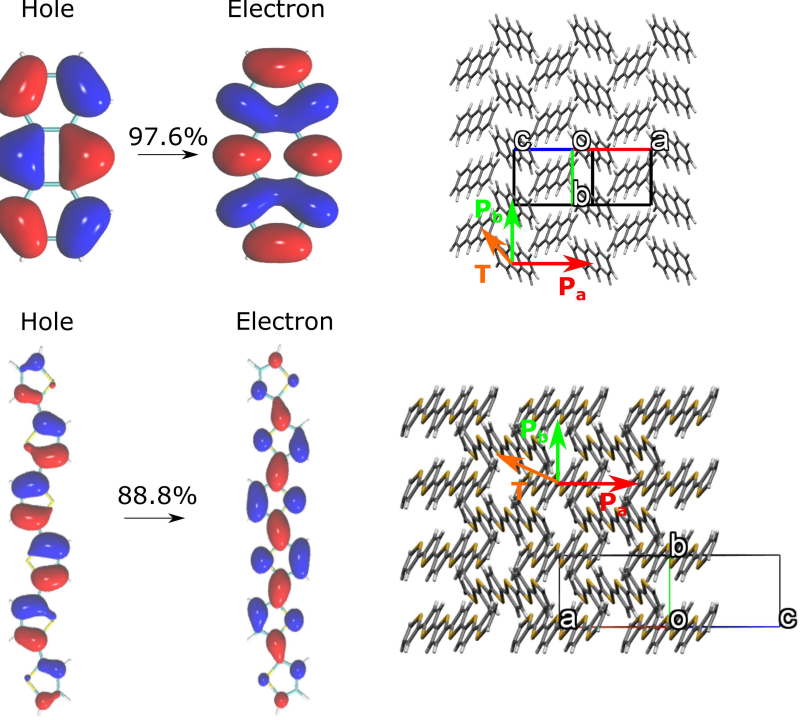

Hole
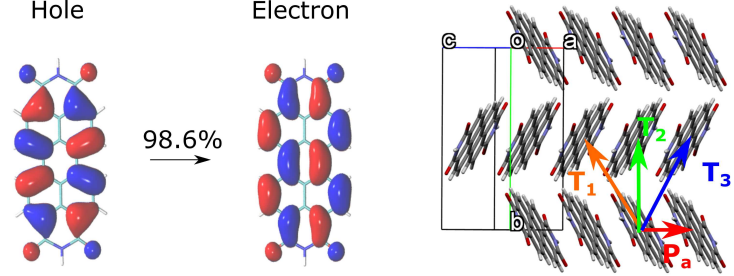

Hole
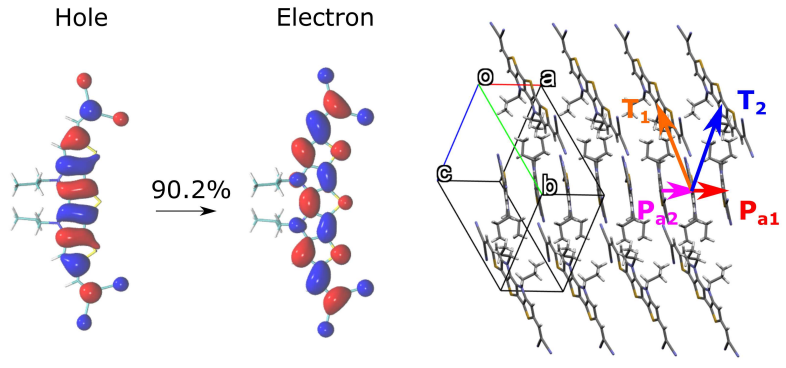

$$
\text { Hole }
$$

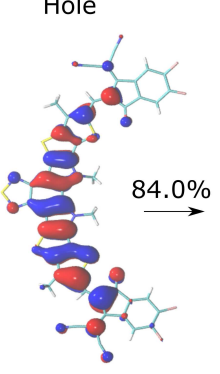

Electron
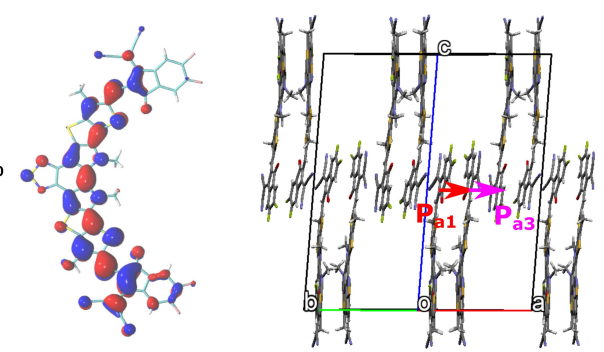

Figure 1: Organic molecular crystals investigated in this work. The chemical structures of the single molecules (left panels), natural transition orbitals (NTOs) for the first singlet excited state (middle panels) and the experimental crystal structure (right panels) are shown for anthracene (ANT, a), $\alpha$-sexithiophene (a6T, b), perylenetetracarboxylic diimide (PDI, c), dicyanovinyl-capped S,Nheteropentacene (DCVSN5, d) and (Y6, e). Directions of strongest excitonic couplings are indicated in the crystal structures and labelled according with their crystallographic direction (e.g. $P_{a}, P_{b}, T$, see also Table 1). In Y6, $P_{a 2}$ is eclipsed by $P_{a 1}$. The unit cell axes $a, b, c$ are shown in red, green and blue. The percentages indicated denote the contribution to the S1 singlet excited states due to the NTOs shown. The NTOs labelled "Hole" and "Electron" resemble very closely the HOMO and LUMO of each molecule. 

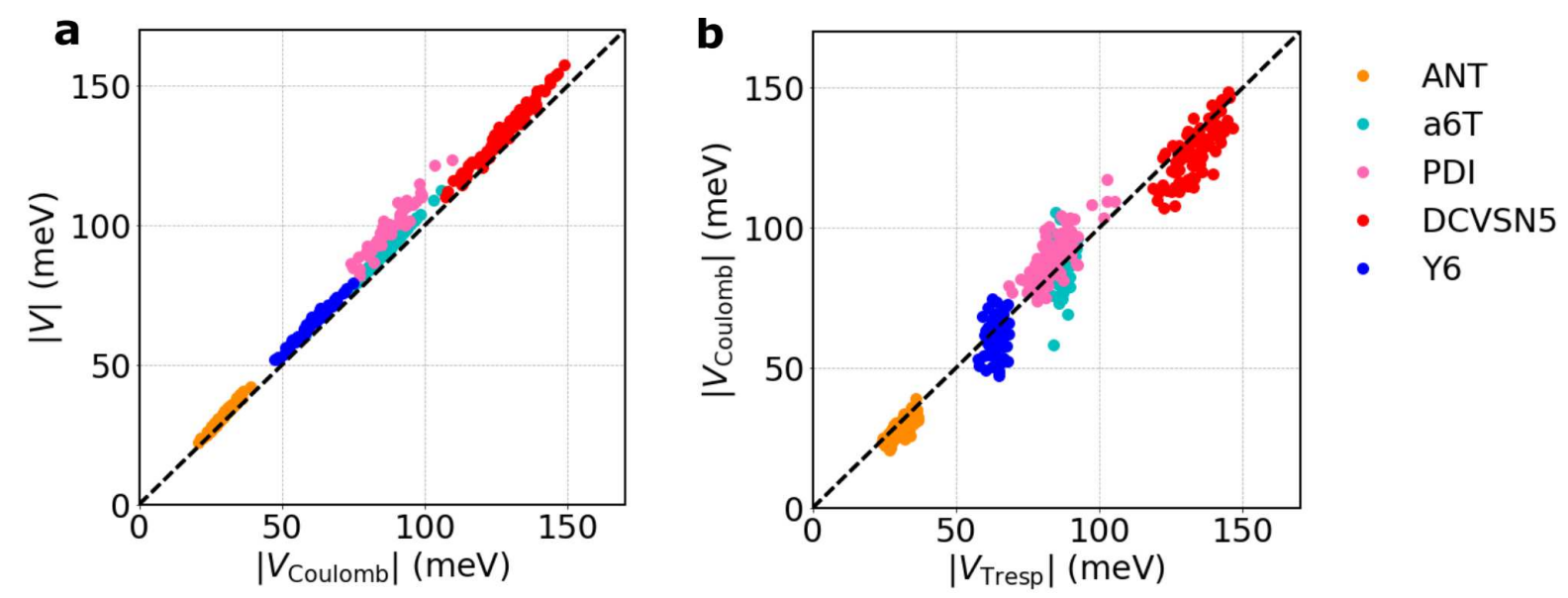

Figure 2: Accuracy of fast excitonic coupling estimators. (a) Correlation between the reference total excitonic coupling $V$, obtained using MS-FED-FCD (Eq. 7 in the Supplementary Information) and $V^{\text {Coulomb }}$ (Eq. 10) for neighbouring molecules along the $\pi$-stacking direction ( $P_{b}$ of ANT and a6T and $P_{a}, P_{a 1}, P_{a 2}$ for PDI, DCVSN5 and Y6 respectively). Excitonic couplings were calculated for configurations taken from MD trajectories generated for supercells of the crystals at $300 \mathrm{~K}$. MS-FED-FCD, $V$, couplings correspond to the off-diagonal block $\mathbb{H}_{\mathrm{XT1} 1 \mathrm{XT2}}^{\prime \prime \prime}$ related to the lowest localized excited states in molecules $k$ and $l$, respectively and the diabatization was performed using the first 20 adiabatic states, which proved to be sufficient for convergence (see Supplementary Information). (b) Correlation between $V^{\text {Coulomb }}$ (Eq. 10) and $V_{\text {TrESP }}$ (Eq. 11). In both panels the mean relative unsigned error is ca. $7 \%$. 


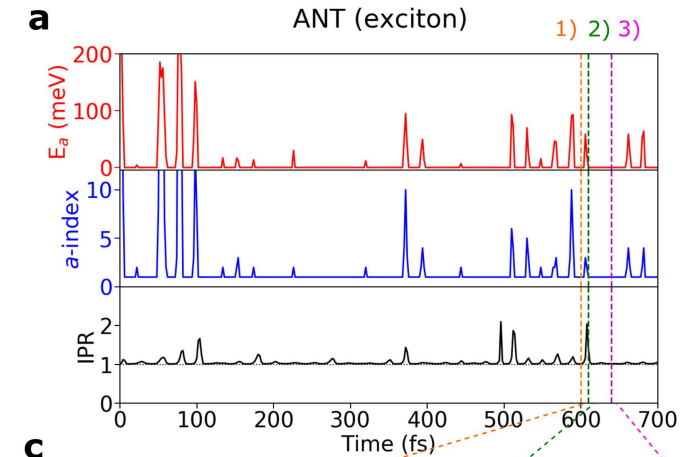

C 1) $\mathrm{t}=600 \mathrm{fs}$
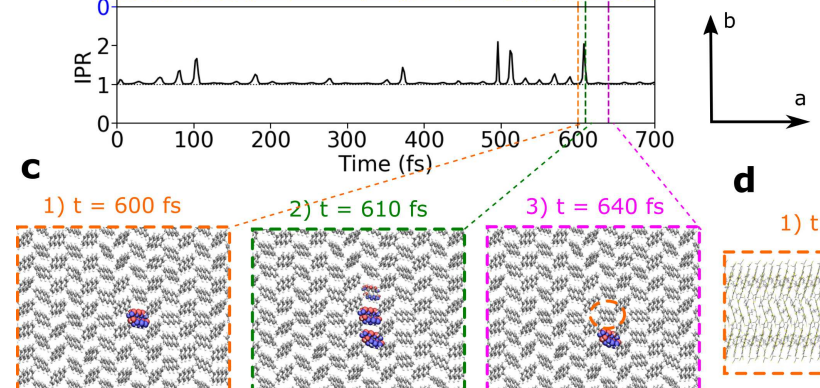

b
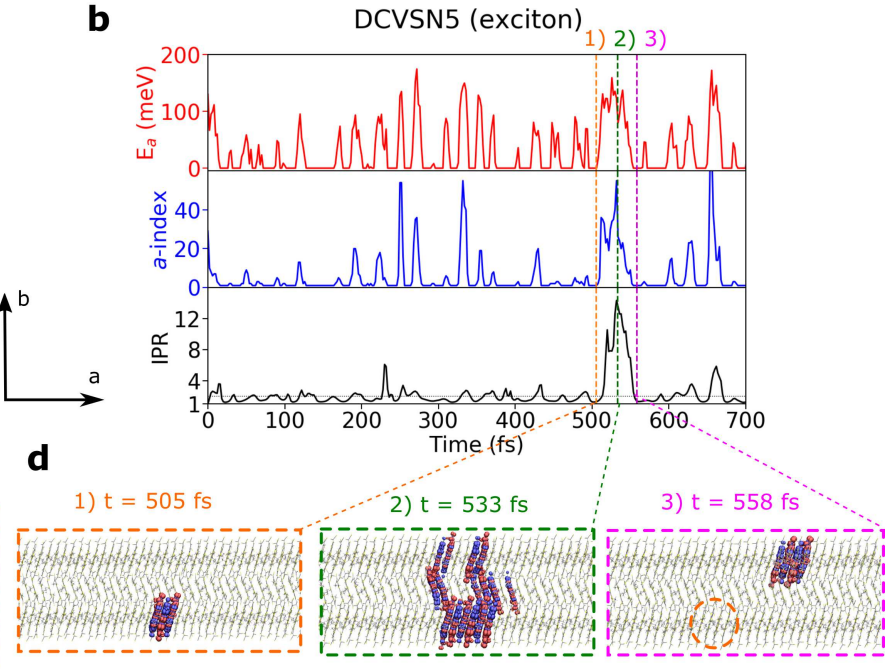

Figure 3: Transient delocalization mechanism of excitons in two of the investigated systems. Panels (a) and (b) show time series along a single representative FE-SH trajectory for ANT and DCVSN5, respectively. Top panels: energy of the active potential energy surface on which the nuclear dynamics is run in FE-SH simulations, $E_{a}$ (red lines), referred to the ground state. Middle panels: index of the active exciton band state, $a$ (blue lines, index $a=0$ corresponds to the ground state which is the bottom of the excitonic band). Bottom panels: IPR of the excitonic wavefunction $\Psi(t)$, Eq. 3, (black lines) and average IPR of $\Psi(t)$ over the swarm of FE-SH trajectories (horizontal dashed grey lines). Note the correlation between the intra-band excitations, i.e. eigenstate index $a$, excitation energy and IPR of $\Psi(t)$. The panels (c) and (d) depict a representative "transient delocalization event" of the excitonic wavefunction $\Psi(t)$ resulting in wavefunction displacement and diffusion. $\Psi(t)$ (Eq. 3) is represented by isosurfaces of the transition density on each molecule scaled by the expansion coefficient $u_{l}$. The transition density is approximated by the conjugated product of HOMO and LUMO orbitals. 
a

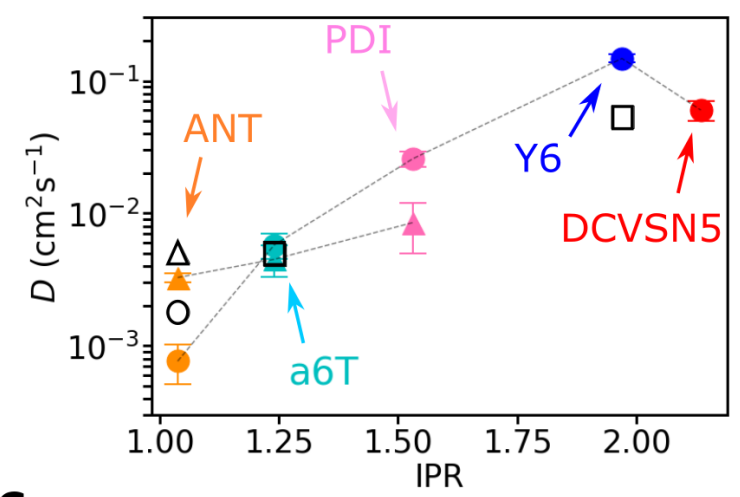

C

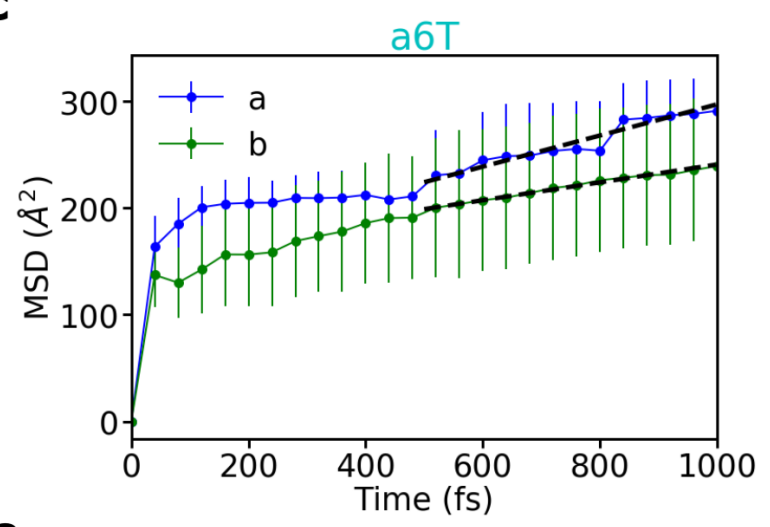

e

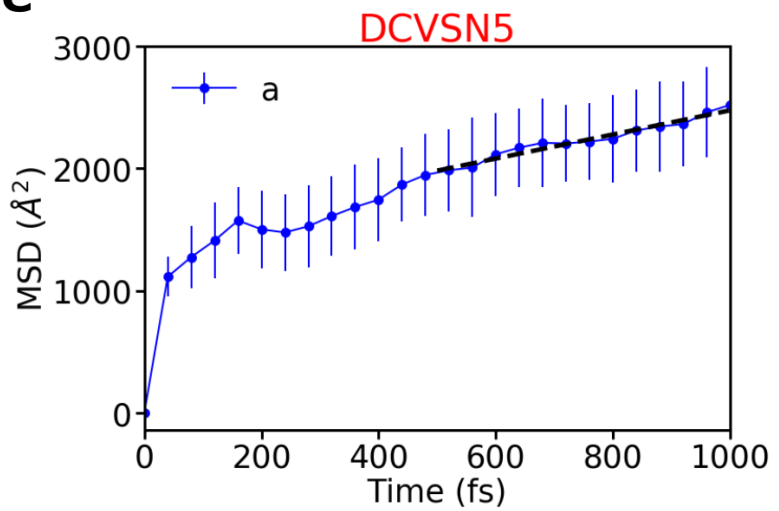

b

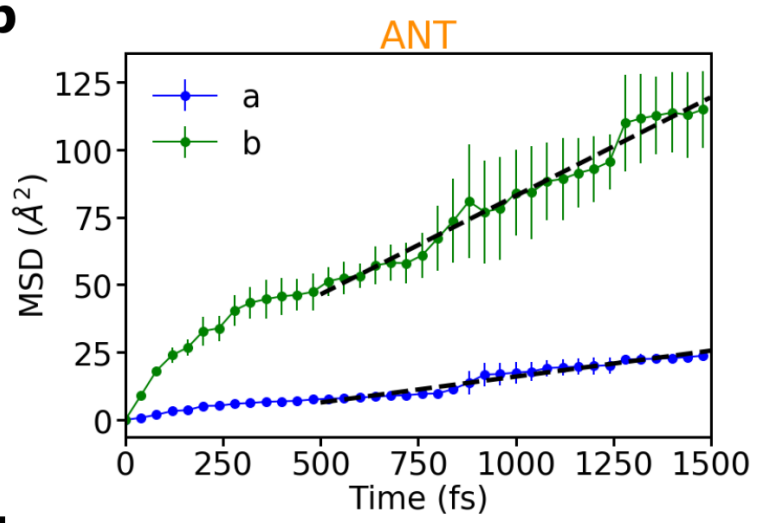

d

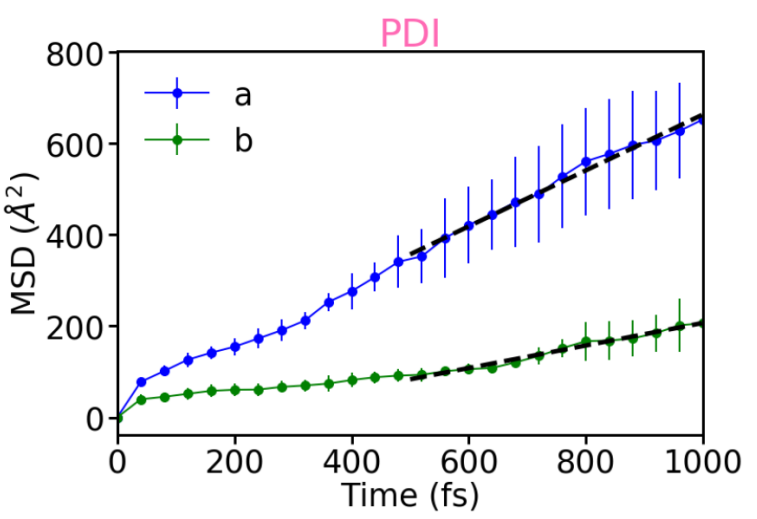

$\mathbf{f}$

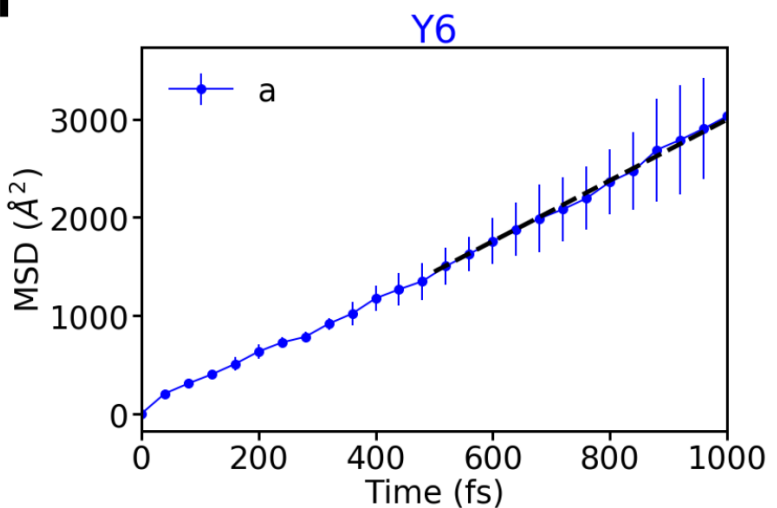

Figure 4: Mean squared displacement (MSD), exciton diffusion constant $(D)$ and delocalization of the excitonic wavefunction. (a) Exciton diffusion constant against inverse participation ratio (IPR) for the system investigated (different colors are used for computed values). a direction is represented by filled circles, while $b$ with filled up triangles where available. The diffusion constants and IPR were averaged over FE-SH simulations carried out for different system sizes, as reported in Supplementary Figure 8. Errors bars indicate the corresponding standard deviations. The black empty symbols are experimental diffusion constant estimates found as discussed in Table 2 and positioned at the calculated IPR (as this is not available from experiments). When the crystallographic direction is not specified from experiments we used empty black squares. Notably $D$ spans more than 2 order of magnitude going from ANT to DCVSN5 systems. (b-f) Representative MSD for system sizes as given in Methods. The MSDs along a and $b$ crystallographic directions (blue and green, respectively) are obtained from the average of 600 FE-SH trajectories. Error bars are obtained by block-averaging over 3 blocks, 200 trajectories each. The diffusion constant is obtained from linear fits to the linear MSD portion (dashed black lines). 

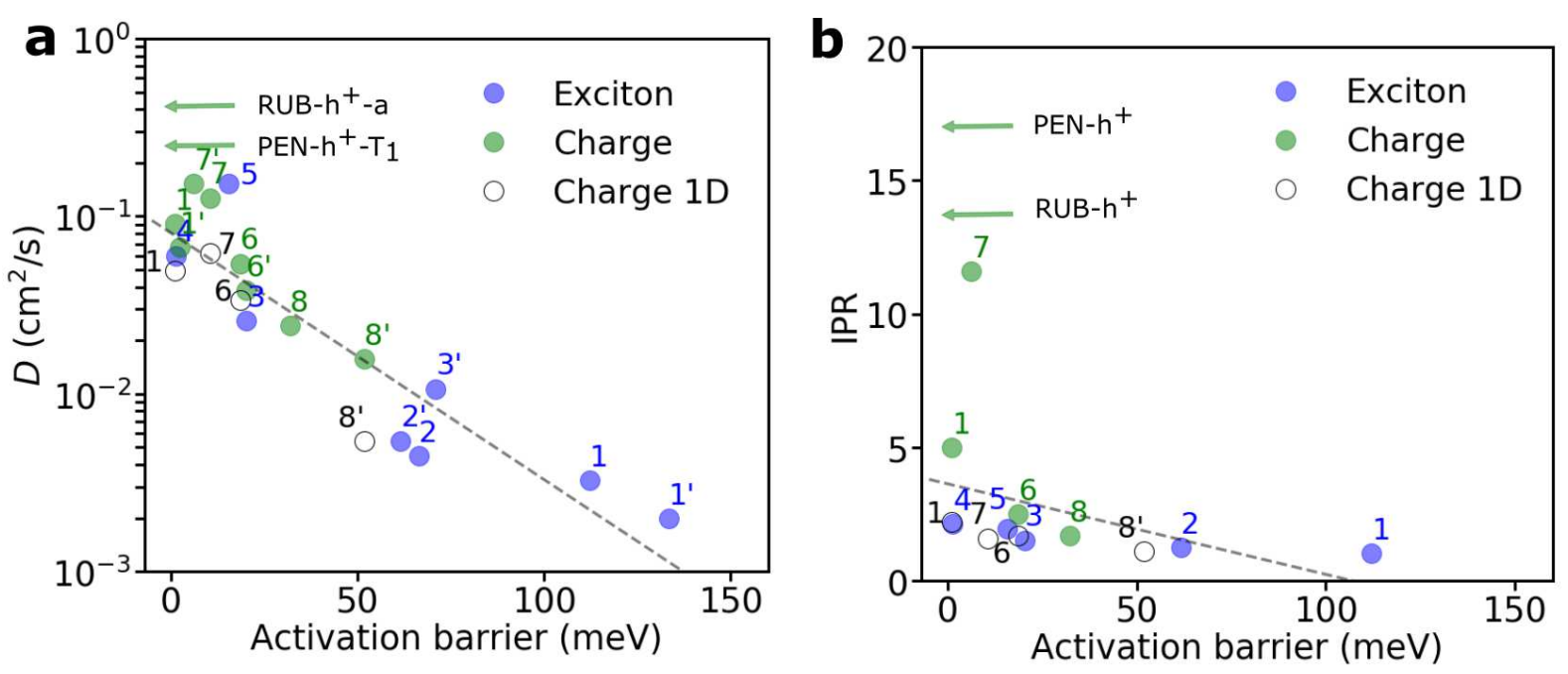

Figure 5: Comparison charge vs exciton transport. Correlation between (a) the diffusion constant ( $D$, Eq. 4) and (b) the IPR (Eq. 8) of charges and excitons against the activation barrier (Eq. 1) for different systems, respectively. Data from present exciton transport simulations are depicted in blue (ANT (1), a6T (2), PDI (3), DCVSN5 (4) and Y6 (5)). Data for charge transport simulations for the 2D conductive layers of OSs are taken from Ref. ${ }^{9}$ and depicted in green (ANT (1), naphthalene (6), perylene (7), pMSB (8), rubrene (RUB) and pentacene (PEN)). For 2D simulations, 2 data points are shown for diffusion constant and activation barrier, one for the direction of highest coupling (unprimed number) and one for the direction of second highest coupling (primed number). For comparison, 1D charge transport simulations taken from Ref. ${ }^{8}$ are indicated by empty black circles and the values reported along the chain direction. The best linear fit to the data is indicated by a dashed line. 
Table 1: Computed parameters for exciton transport in the molecular OSs shown in Figure 1. All values in $\mathrm{meV}^{a}$

\begin{tabular}{ccccccccc} 
& & Dist. & $V_{k l} b, c$ & $V_{k l}^{\text {Coulomb } b}$ & $V_{k l}^{\operatorname{TrESP} d}$ & $\left\langle V_{k l}^{\operatorname{TrESP}}\right\rangle^{e}$ & $\sigma_{k l}^{\operatorname{TrESP}} f$ & $\lambda^{\mathrm{XT}} g$ \\
\hline \multirow{4}{*}{ ANT } & $P_{b}$ & 6.04 & -27.88 & -25.95 & -26.34 & -29.46 & 3.17 & \\
& $T$ & 5.24 & 6.40 & 4.53 & 4.47 & 4.34 & 5.27 & 560.86 \\
& $P_{a}$ & 8.56 & 4.35 & 4.26 & 4.17 & 4.50 & 0.79 & \\
\hline \multirow{4}{*}{ a6T } & $P_{b}$ & 5.68 & 91.92 & 88.01 & 86.89 & 86.46 & 2.68 & \\
& $T$ & 5.38 & -101.15 & -96.14 & -95.45 & -93.55 & 3.40 & 558.11 \\
& $P_{a}$ & 9.14 & 42.01 & 41.83 & 41.60 & 40.64 & 2.00 & \\
\hline \multirow{3}{*}{ PDI } & $P_{a}$ & 4.87 & 100.06 & 85.74 & 85.46 & 105.74 & 8.38 & \\
& $T_{1}$ & 9.47 & -31.97 & -31.88 & -31.84 & -28.68 & 1.38 & \multirow{2}{*}{390.47} \\
& $T_{2}$ & 9.40 & -17.89 & -17.05 & -17.09 & -12.94 & 2.85 & \\
\hline \multirow{4}{*}{ DCVSN5 } & $P_{a 1}$ & 3.64 & -137.65 & -135.61 & -134.97 & -131.76 & 6.08 & \\
& $P_{a 2}$ & 4.46 & -151.93 & -141.59 & -142.04 & -139.16 & 7.93 & 320.09 \\
& $T_{1}$ & 14.20 & 24.14 & 24.36 & 24.29 & 22.41 & 2.89 & \\
\hline \multirow{2}{*}{ Y6 } & $P_{a 1}$ & 9.41 & -86.38 & -73.67 & -63.15 & -62.25 & 3.33 & \\
& $P_{a 2}$ & 15.35 & -61.26 & -55.16 & -63.26 & -61.90 & 4.49 & 249.71 \\
& $P_{a 3}$ & 18.34 & 52.58 & 47.44 & 47.47 & 39.77 & 4.99 & \\
\hline
\end{tabular}

${ }^{a}$ Excitonic couplings (meV) are computed for few of the closest crystal pairs. Basis set is fixed to 6-31g(d,p). The choice of the DFT functional is discussed in the main text and in the Supplementary Information. ${ }^{b}$ The sign of $V_{k l}$ and $V_{k l}^{\text {Coulomb }}$ (Eq. 10) is adjusted according to the sign obtained from $V_{k l}^{\text {TrESP }}$ (Eq. 11), where a consistent phase of the transition density on all the molecules is given by construction. A negative coupling does not necessarily mean $\mathrm{J}$-aggregation in this context as the coupling sign depends on the relative orientation of the interacting transition dipoles (see also Supplementary Fig. 6). ${ }^{c} V_{k l}$ couplings are evaluated using MS-FED-FCD diabatization approach (using the first 20 excited states for the diabatization procedure, see main text and Supplementary Information). ${ }^{d}$ TrESP are parametrized as described in Methods and using the level of theory mentioned above. ${ }^{e}$ Mean excitonic couplings are averaged over values extracted from all the pairs (in a given direction) for all the time snapshots of $10 \mathrm{FE}-\mathrm{SH}$ trajectories of at least 1 ps. ${ }^{f}$ Fluctuations of electronic couplings from the aformentioned trajectories, $\sigma_{k l}^{\text {TrESP }}=\sqrt{\left\langle\left(H_{k l}^{\text {TrESP }}-\left\langle H_{k l}^{\text {TrESP }}\right\rangle\right)^{2}\right\rangle} .{ }^{g}$ Reorganization energies (meV) corresponding to the S1 state, $\lambda^{\mathrm{XT}}$, are computed with Eq. 12 and the same level of theory used for coupling calculations. 
Table 2: Wavefunction delocalization (IPR), exciton diffusion constants $(D)$ and exciton diffusion lengths $(L)$.

\begin{tabular}{|c|c|c|c|c|c|c|c|}
\hline \multirow[b]{2}{*}{ Systems } & \multirow[b]{2}{*}{ Dir. } & \multicolumn{3}{|c|}{ FE-SH } & \multicolumn{3}{|c|}{ Experiment } \\
\hline & & IPR & $D\left(10^{-3} \mathrm{~cm}^{2} \mathrm{~s}^{-1}\right)^{a}$ & $L(\mathrm{~nm})^{b}$ & $\tau_{\exp }(\mathrm{s})$ & $D_{\exp }\left(10^{-3} \mathrm{~cm}^{2} \mathrm{~s}^{-1}\right)^{c}$ & $L_{\text {exp }}(\mathrm{nm})$ \\
\hline \multirow[b]{2}{*}{ ANT } & $a$ & & $0.77 \pm 0.25$ & 39 & \multirow{2}{*}{$1.0 \times 10^{-8 d}$} & $1.8^{e}$ & $60^{f}$ \\
\hline & $b$ & 1.0 & $3.3 \pm 0.77$ & 81 & & $5.0^{e}$ & $100^{f}$ \\
\hline a6T & $a$ & 12 & $5.8 \pm 1.2$ & 46 & $1.8 \times 10^{-9} \mathrm{~g}$ & $4.9^{h}$ & $60^{i}$ \\
\hline \multirow[b]{2}{*}{ PDI } & $a$ & \multirow[b]{2}{*}{1.5} & $26 \pm 3.5$ & - & - & - & - \\
\hline & $b$ & & $8.5 \pm 3.0$ & - & - & - & - \\
\hline DCVSN5 & $a$ & 2.1 & $60 \pm 11$ & - & - & - & - \\
\hline Y6 & $a$ & 2.0 & $150 \pm 6.5$ & 86 & $2.5 \times 10^{-10 l}$ & $54^{m}$ & $37^{l}\left(90^{n}\right)$ \\
\hline
\end{tabular}

${ }^{a}$ In FE-SH $D$ is directly computed using Eq. 6. The diffusion constants were averaged over FE-SH simulations carried out for different system sizes, as reported in Supplementary Figure 8. Errors bars indicate the corresponding standard deviations. ${ }^{b} L=\sqrt{2 D \tau_{\exp }}$. ${ }^{c}$ Usually the diffusion constant, $D_{\text {exp }}$, is not directly measured in experiments. In this case, we estimated $D_{\exp }$ using the experimentally observed $L_{\exp } .{ }^{d}$ Taken from Ref. ${ }^{27} e$ Estimated considering $D_{\exp }=L_{\exp }^{2} / 2 \tau_{\exp }$ assuming that the transport occur in different $1 \mathrm{D}$ directions as done in Ref. ${ }^{30}$. ${ }^{f}$ Taken from Ref. ${ }^{28,29}$. ${ }^{g}$ Taken from Ref. ${ }^{69}{ }^{h}$ Estimated assuming that a6T forms a 2D thin film and the diffusion occurs isotropically within the herringbone plane, so that $D_{\exp }=L_{\exp }^{2} / 4 \tau_{\exp } \cdot{ }^{i}$ Taken from Ref. ${ }^{70}$. Note that $L_{\exp }$ in this case refer to a6T thin film morphology and it should be taken as indicative only (see Supplementary Information for a discussion). ${ }^{l}$ Taken from Ref. ${ }^{5} . L_{\text {exp }}$ in this case refer to Y6 thin film morphology and it should be taken as indicative only. ${ }^{m}$ Taken from Ref. ${ }^{5}$, which estimated this value using a 3D model. ${ }^{n}$ Estimated assuming isotropic exciton transport in 3D and taking $D_{\exp }$ from Ref. ${ }^{5}$. In this case $L_{\exp }=\sqrt{6 D_{\exp } \tau_{\exp }}$. 


\section{References}

[1] Frenkel, J. On the transformation of light into heat in solids. ii. Phys. Rev. 37, 1276 (1931).

[2] Pope, M. \& Swenberg, C. E. Electronic processes in organic crystals and polymers (Oxford University Press on Demand, 1999).

[3] Azzouzi, M., Kirchartz, T. \& Nelson, J. Factors Controlling Open-Circuit Voltage Losses in Organic Solar Cells. Trends Chem. 1, 49-62 (2019).

[4] Mikhnenko, O. V., Blom, P. W. M. \& Nguyen, T.-Q. Exciton diffusion in organic semiconductors. Energy Environ. Sci. 8, 1867-1888 (2015).

[5] Firdaus, Y. et al. Long-range exciton diffusion in molecular non-fullerene acceptors. Nat. Commun. 11, 5220 (2020).

[6] Hestand, N. J. \& Spano, F. C. Expanded Theory of H- and J-Molecular Aggregates: The Effects of Vibronic Coupling and Intermolecular Charge Transfer. Chem. Rev. 118, 7069-7163 (2018).

[7] Nematiaram, T., Padula, D. \& Troisi, A. Bright Frenkel Excitons in Molecular Crystals: A Survey. Chem. Mater. 33, 3368-3378 (2021).

[8] Giannini, S. et al. Quantum localization and delocalization of charge carriers in organic semiconducting crystals. Nat. Comm. 10, 3843 (2019).

[9] Giannini, S., Ziogos, O. G., Carof, A., Ellis, M. \& Blumberger, J. Flickering Polarons Extending over Ten Nanometres Mediate Charge Transport in High-Mobility Organic Crystals. Adv. Theory Simul. 3, 2000093 (2020).

[10] Balzer, D., Smolders, T. J. A. M., Blyth, D., Hood, S. N. \& Kassal, I. Delocalised kinetic Monte Carlo for simulating delocalisation-enhanced charge and exciton transport in disordered materials. Chem. Sci 12, 2276-2285 (2021).

[11] Stehr, V., Fink, R. F., Engels, B., Pflaum, J. \& Deibel, C. Singlet exciton diffusion in organic crystals based on marcus transfer rates. J. Chem. Theory Comput. 10, 1242-1255 (2014).

[12] Hume, P. A., Jiao, W. \& Hodgkiss, J. M. Long-range exciton diffusion in a non-fullerene acceptor: approaching the incoherent limit. J. Mater. Chem. C 9, 1419-1428 (2021).

[13] Popp, W., Brey, D., Binder, R. \& Burghardt, I. Quantum Dynamics of Exciton Transport and Dissociation in Multichromophoric Systems. Ann. Rev. Phys. Chem. 72, 591-616 (2021). 
[14] Gil, E. S., Granucci, G. \& Persico, M. Surface Hopping Dynamics with the Frenkel Exciton Model in a Semiempirical Framework. J. Chem. Theory Comput. 17, 7373-7383 (2021).

[15] Menger, M. F. S. J., Plasser, F., Mennucci, B. \& González, L. Surface Hopping within an Exciton Picture. An Electrostatic Embedding Scheme. J. Chem. Theory Comput. 14, 6139-6148 (2018).

[16] Li, X., Parrish, R. M., Liu, F., Kokkila Schumacher, S. I. \& Martínez, T. J. An Ab Initio Exciton Model Including Charge-Transfer Excited States. J. Chem. Theory Comput. 13, 3493-3504 (2017).

[17] Spencer, J., Gajdos, F. \& Blumberger, J. Fob-sh: Fragment orbital-based surface hopping for charge carrier transport in organic and biological molecules and materials. J. Chem. Phys. 145, 064102 (2016).

[18] Carof, A., Giannini, S. \& Blumberger, J. How to calculate charge mobility in molecular materials from surface hopping non-adiabatic molecular dynamics - beyond the hopping/band paradigm. Phys. Chem. Chem. Phys. 21, 26368-26386 (2019).

[19] Giannini, S., Carof, A., Ellis, M., Ziogos, O. G. \& Blumberger, J. From atomic orbitals to nano-scale charge transport with mixed quantum/classical non-adiabatic dynamics: Method, implementation and application. In Multiscale Dynamics Simulations: Nano- and Nano-bio Systems in Complex Environments, chap. 6 .

[20] Sneyd, A. J. et al. Efficient energy transport in an organic semiconductor mediated by transient exciton delocalization. Sci. Adv. 7, eabh4232 (2021).

[21] Prodhan, S., Giannini, S., Wang, L. \& Beljonne, D. Long-range interactions boost singlet exciton diffusion in nanofibers of $\pi$-extended polymer chains. J. Phys. Chem. Let. 12, 8188-8193 (2021).

[22] Mason, R. The crystallography of anthracene at $95 \mathrm{k}$ and $290 \mathrm{k}$. Acta Crystallogr. 17, 547-555 (1964).

[23] Siegrist, T. et al. The crystal structure of the high-temperature polymorph of $\alpha$-hexathienyl ( $\alpha-$ 6t/ht). Mater. Res. 10, 2170-2173 (1995).

[24] Tojo, K. \& Mizuguchi, J. Refinement of the crystal structure of 3, 4: 9, 10-perylenebis (dicarboximide), c24h10n2o4, at 263 k. Zeitschrift für Kristallographie-New Crystal Structures 217, 45-46 (2002).

[25] Mishra, A. et al. A-D-A-type S , N -Heteropentacenes: Next-Generation Molecular Donor Materials for Efficient Vacuum-Processed Organic Solar Cells. Adv. Mater. 26, 7217-7223 (2014).

[26] Xiao, C., Li, C., Liu, F., Zhang, L. \& Li, W. Single-crystal field-effect transistors based on a fused-ring electron acceptor with high ambipolar mobilities. J. Mater. Chem. C 8, 5370-5374 (2020). 
[27] Powell, R. C. \& Soos, Z. G. Singlet exciton energy transfer in organic solids. Journal of Luminescence 11, 1-45 (1975).

[28] Mulder, B. Anisotropy of light absorption and exciton diffusion in anthracene crystals determined from externally sensitized fluorescence. Philips Res. Rep 22, 142-149 (1967).

[29] Mulder, B. Symmetry of the fluorescence and absorption spectra of anthracene crystals. J. Phys. Chem. Solids 29, 182-184 (1968).

[30] Aragó, J. \& Troisi, A. Regimes of exciton transport in molecular crystals in the presence of dynamic disorder. Adv. Funct. Mater. 26, 2316-2325 (2016).

[31] Kranz, J. J. \& Elstner, M. Simulation of Singlet Exciton Diffusion in Bulk Organic Materials. J. Chem. Theory Comput. 12, 4209-4221 (2016).

[32] Dong, Y. et al. Orientation dependent molecular electrostatics drives efficient charge generation in homojunction organic solar cells. Nat. Commun. 11, 4617 (2020).

[33] Mishra, A. \& Bäuerle, P. Small molecule organic semiconductors on the move: promises for future solar energy technology. Angew. Chem. Int. Ed. 51, 2020-2067 (2012).

[34] Hou, J., Inganäs, O., Friend, R. H. \& Gao, F. Organic solar cells based on non-fullerene acceptors. Nat. Mater. 17, 119-128 (2018).

[35] Pandya, R. et al. Femtosecond Transient Absorption Microscopy of Singlet Exciton Motion in SideChain Engineered Perylene-Diimide Thin Films. J. Phys. Chem. A 124, 2721-2730 (2020).

[36] Li, C. et al. Non-fullerene acceptors with branched side chains and improved molecular packing to exceed 18\% efficiency in organic solar cells. Nature Energy 6, 605-613 (2021).

[37] Cui, Y. et al. Single-Junction Organic Photovoltaic Cell with 19\% Efficiency. Advanced Materials 33 (2021).

[38] Kenny, E. P. \& Kassal, I. Benchmarking Calculations of Excitonic Couplings between Bacteriochlorophylls. J. Phys. Chem. B 120, 25-32 (2016).

[39] Curutchet, C. \& Mennucci, B. Quantum Chemical Studies of Light Harvesting. Chem. Rev. 117, 294-343 (2017).

[40] Cupellini, L., Corbella, M., Mennucci, B. \& Curutchet, C. Electronic energy transfer in biomacromolecules. Wiley Interdiscip. Rev. Comput. Mol. Sci. 9, 1-23 (2019). 
[41] Madjet, M., Abdurahman, A. \& Renger, T. Intermolecular coulomb couplings from ab initio electrostatic potentials: application to optical transitions of strongly coupled pigments in photosynthetic antennae and reaction centers. J. Phys. Chem. B 110, 17268-17281 (2006).

[42] Cupellini, L., Giannini, S. \& Mennucci, B. Electron and excitation energy transfers in covalently linked donor-acceptor dyads: mechanisms and dynamics revealed using quantum chemistry. Phys. Chem. Chem. Phys 20, 395-403 (2018).

[43] Nottoli, M. et al. The role of charge-transfer states in the spectral tuning of antenna complexes of purple bacteria. Photosynth. Res. 137, 215-226 (2018).

[44] Tölle, J., Cupellini, L., Mennucci, B. \& Neugebauer, J. Electronic couplings for photo-induced processes from subsystem time-dependent density-functional theory: The role of the diabatization. J. Chem. Phys. 153, 184113 (2020).

[45] Carof, A., Giannini, S. \& Blumberger, J. Detailed balance, internal consistency and energy conservation in fragment orbital-based surface hopping. J. Chem. Phys. 147, 214113 (2017).

[46] Bai, X., Qiu, J. \& Wang, L. An efficient solution to the decoherence enhanced trivial crossing problem in surface hopping. J. Chem. Phys. 148, 104106 (2018).

[47] Ellis, M., Yang, H., Giannini, S., Ziogos, O. G. \& Blumberger, J. Impact of nanoscale morphology on charge carrier delocalization and mobility in an organic semiconductor. Adv. Mat. 2104852 (2021).

[48] Yamagata, H. et al. The nature of singlet excitons in oligoacene molecular crystals. J. Chem. Phys. 134, 204703 (2011).

[49] Spencer, J., Scalfi, L., Carof, A. \& Blumberger, J. Confronting surface hopping molecular dynamics with marcus theory for a molecular donor-acceptor system. Faraday Discuss. 195, 215-236 (2016).

[50] Oberhofer, H., Reuter, K. \& Blumberger, J. Charge transport in molecular materials: an assessment of computational methods. Chem. Rev. 117, 10319-10357 (2017).

[51] Fratini, S., Ciuchi, S., Mayou, D., Laissardiere, G. T. d. \& Troisi, A. A map of high-mobility molecular semiconductors. Nat. Mater. 16, 998-1002 (2017).

[52] Fratini, S., Nikolka, M., Salleo, A., Schweicher, G. \& Sirringhaus, H. Charge transport in highmobility conjugated polymers and molecular semiconductors. Nat. Mater. 19, 491-502 (2020).

[53] Stojanovic, L. \& Crespo-Otero, R. Aggregation-induced emission in the tetraphenylthiophene crystal: The role of triplet states. J. Phys. Chem. C 124, 17752-17761 (2020).

[54] Tully, J. C. Molecular dynamics with electronic transitions. J. Chem. Phys. 93, 1061-1071 (1990). 
[55] Giannini, S., Carof, A. \& Blumberger, J. Crossover from hopping to band-like charge transport in an organic semiconductor model: Atomistic non-adiabatic molecular dynamics simulation. J. Phys. Chem. Lett. 9, 3116-3123 (2018).

[56] Yanai, T., Tew, D. P. \& Handy, N. C. A new hybrid exchange-correlation functional using the coulomb-attenuating method (cam-b3lyp). Chem. Phys. letters 393, 51-57 (2004).

[57] Chai, J.-D. \& Head-Gordon, M. Long-range corrected hybrid density functionals with damped atomatom dispersion corrections. Phys. Chem. Chem. Phys 10, 6615-6620 (2008).

[58] Yi, Y., Coropceanu, V. \& Brédas, J.-L. A comparative theoretical study of exciton-dissociation and charge-recombination processes in oligothiophene/fullerene and oligothiophene/perylenediimide complexes for organic solar cells. J. Mater. Chem 21, 1479 (2011).

[59] Carmen Ruiz Delgado, M., Kim, E. G., Da Silva Filho, D. A. \& Bredas, J. L. Tuning the chargetransport parameters of perylene diimide single crystals via end and/or core functionalization: A density functional theory investigation. J. Am. Chem. Soc. 132, 3375-3387 (2010).

[60] Frisch, M. J. et al. (Gaussian 16, Revision C.01, Gaussian Inc., Wallingford, CT, 2016).

[61] Yang, C.-H. \& Hsu, C.-P. A multi-state fragment charge difference approach for diabatic states in electron transfer: Extension and automation. J. Chem. Phys. 139, 154104 (2013).

[62] Dexter, D. L. A theory of sensitized luminescence in solids. J. Chem. Phys. 21, 836-850 (1953).

[63] Iozzi, M. F., Mennucci, B., Tomasi, J. \& Cammi, R. Excitation energy transfer (eet) between molecules in condensed matter: A novel application of the polarizable continuum model $(\mathrm{pcm})$. J. Chem. Phys. 120, 7029-7040 (2004).

[64] Stehr, V., Fink, R. F., Tafipolski, M., Deibel, C. \& Engels, B. Comparison of different rate constant expressions for the prediction of charge and energy transport in oligoacenes. Wiley Interdisciplinary Reviews: Computational Molecular Science 6, 694-720 (2016).

[65] Case, D. A. et al. et al. (AMBER 10, University of California, San Francisco, 2008).

[66] Kamencek, T. et al. Evaluating Computational Shortcuts in Supercell-Based Phonon Calculations of Molecular Crystals: The Instructive Case of Naphthalene. J. Chem. Theory Comput. 16, 2716-2735 (2020).

[67] Landi, A. \& Padula, D. Multiple charge separation pathways in new-generation non-fullerene acceptors: a computational study. J. Mater. Chem. A 9, 24849-24856 (2021). 
[68] Hutter, J., Iannuzzi, M., Schiffmann, F. \& VandeVondele, J. cp2k: atomistic simulations of condensed matter systems. Wiley Interdiscip. Rev.: Comput. Mol. Sci. 4, 15-25 (2014).

[69] Horowitz, G., Valat, P., Garnier, F., Kouki, F. \& Wintgens, V. Photoinduced spontaneous and stimulated emission in sexithiophene single crystals. Optical Materials 9, 46-52 (1998).

[70] Mani, A., Schoonman, J. \& Goossens, A. Photoluminescence Study of Sexithiophene Thin Films. J. Phys. Chem. B 109, 4829-4836 (2005). 


\section{Supplementary Files}

This is a list of supplementary files associated with this preprint. Click to download.

- Slnew.pdf 